\title{
Virtual Machine Consolidated Placement Based on Multi-Objective Biogeography-Based Optimization
}

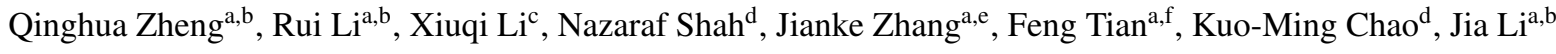 \\ ${ }^{a}$ MOE Key Lab for Intelligent Networks and Network Security, Xi'an Jiaotong University, Xi'an, China; \\ ${ }^{b}$ Department of Computer Science and Technology, Xi'an Jiaotong University, Xi'an, China; \\ ${ }^{c}$ Department of Computer Science and Mathematics, University of North Carolina at Pembroke, Pembroke, NC 28372, USA; \\ ${ }^{d}$ Faculty of Engineering and Computing, Coventry University, UK; \\ ${ }^{e}$ School of Science, Xi' an University of Posts and Telecommunications, Xi'an 710121, China; \\ ${ }^{f}$ Systems Engineering Institute, Xi'an Jiaotong University, Xi'an, China;
}

\begin{abstract}
Virtual machine placement (VMP) is an important issue in selecting most suitable set of physical machines (PMs) for a set of virtual machines (VMs) in cloud computing environment. VMP problem consists of two sub problems: incremental placement $(\mathrm{VMiP})$ problem and consolidated placement $(\mathrm{VMcP})$ problem. The goal of $\mathrm{VMcP}$ is to consolidate the VMs to more suitable PMs. The challenge in VMcP problem is how to find optimal solution effectively and efficiently especially when VMcP is a kind of NP-hard problem. In this paper, we present a novel solution to the VMcP problem called VMPMBBO. The proposed VMPMBBO treats VMcP problem as a complex system and utilizes the biogeography-based optimization (BBO) technique to optimize the virtual machine placement that minimizes both the resource wastage and the power consumption at the same time. Extensive experiments have been conducted using synthetic data from related literature and data from two real datasets. First of all, the necessity of VMcP has been proved by experimental results obtained by applying VMPMBBO. Then, the proposed method is compared with two existing multi-objective VMcP optimization algorithms and it is shown that VMPMBBO has better convergence characteristics and is more computationally efficient as well as robust. And then, the issue of parameter setting of the proposed method has been discussed. Finally, adaptability and extensibility of VMPMBBO have also been proved through experimental results. To the best of our knowledge, this work is the first approach that applies biogeography-based optimization (BBO) to virtual machine placement.
\end{abstract}

Keywords: Virtual machine placement, Multi-objective optimization, Resource utilization, Biogeography-based optimization, Cloud computing

\section{Introduction}

Cloud computing has been a popular computing paradigm in the IT industry since 2008. It delivers computing infrastructures, computing platforms, and software as hosted services on demand over the Internet. Cloud users can access computing resources without having to own, manage, and maintain them. There are three common cloud computing models known as Infrastructure-as-a-Service (IaaS), Platform-as-a-Service (PaaS), and Software-as-a-Service (SaaS) $[1,2,3]$. In the most basic model IaaS, like Amazon Web Services, cloud users are provided with physical or, more frequently, virtual machines and additional resources like raw block storage, which are allocated from massive computing resource pools in large-scale data centers. The focus of this paper is on IaaS model.

From the perspective of a cloud provider, to reduce the operating cost, the use of computing resources in cloud needs to be maximized. In addition, the power consumption needs to be minimized as it has become a significant contributor to the operating cost [4]. The total electricity used by data centers in the US increased about $56 \%$ from 2005 to 2010 [5].

The core technology in cloud computing is virtualization [6], which separates resources and services from the underlying physical delivery environment. The resources of a single physical machine (PM) are sliced into multiple isolated execution environments for multiple virtual machines (VMs). Virtual Machine Placement (VMP) is an important topic in cloud environment virtualization, in particular in IaaS model. VMP maps a set of virtual machines to 
a set of physical machines. For the cloud providers, a good VMP solution should maximize resource utilization and minimize power consumption.

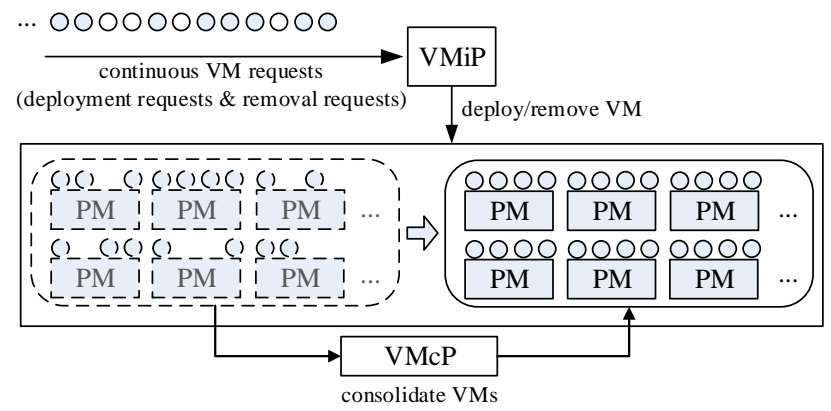

Fig. 1: An example of VMiP and VMcP

The problem of VMP consists of two sub problems: incremental placement (VMiP) problem and consolidated placement (VMcP) problem [7], as shown in Fig. 1. VMiP deals with continuous arrival of VM deployment and removal requests at runtime. Quick response is a crucial metric for ensuring a high service quality of the VMiP. The problem of VMiP has received much attention $[7,8,9,10]$. However, as VMs are being continuously removed over time, this may leads to a situation where infrastructure may be brought to a poor VMs distribution state over a long period of time. Therefore, there is an urgent need for periodically redeploying existing VMs on more suitable servers $[7,11]$. The terms "physical machine" and "server" will be used interchangeably in this paper

$\mathrm{VMcP}$ is used by data centers to increase resource utilization and reduce electric power consumption costs [11]. $\mathrm{VMcP}$ is particularly important when users' workloads are unpredictable and VMcP need to be revisited periodically [11]. Whenever VM instances change, VMs can be relocated and migrated to different physical servers if necessary [11]. VMcP can be invoked periodically, or be triggered based on some preset conditions. The problem of VMcP is NP-hard $[12,13,14,15,16,17]$. The challenge in VMcP problem is how to find optimal solution effectively and efficiently.

The existing research in $\mathrm{VMcP}$ can be classified into five categories based on the techniques being used: heuristic bin packing $[18,19,20,21,22,23,24,25,12,26]$, biology-based optimization $[27,28,29,30,31,32]$, linear programming [33, 34], constraint programming [35], and simulated annealing optimization [36]. Another type of classification is single-objective or multi-objective, based on the number of objectives to be optimized during the placement. Recent research [5, 31, 37] focuses on multi-objective solutions. Both [5] and [31] optimize resource utilization and power consumption. The thermal dissipation cost is also considered in some research efforts [31]. The work in [37] optimizes CPU utilization, network throughput, and disk I/O rate. Existing biology-based optimization algorithms for VMcP include genetic algorithms [29,5], particle swarm optimization [28] and ant colony optimization $[27,31]$.

In this paper, we propose a novel multi-objective VMcP solution named VMPMBBO. It employs a state-of-theart evolutionary algorithm, biogeography-based optimization (BBO) [38, 39], to find the optimal VM placements that simultaneously minimizes both the resource wastage and the power consumption. Compared with two existing multi-objective evolutionary algorithms [5, 31], VMPMBBO has better convergence characteristics and is more computationally efficient. Extensive simulation results confirm the effectiveness, efficiency and robustness of the proposed approach. Adaptability and extensibility of VMPMBBO have also been proved by experimental results. To the best of our knowledge, this work is the first approach that applies biogeography-based optimization (BBO) and complex system optimization to VMP problem.

The paper is organized as follows. In Section 2, the existing VMcP solutions are reviewed. VM placement problem is formulated in Section 3. Section 4 presents background knowledge of the proposed VMPMBBO approach. The simulation results are presented in Section 4. Section 5 evaluates the effectiveness of the proposed approach. Section 6 proves the adaptability and extensibility of VMPMBBO and finally Section 7 concludes the paper. 


\section{Related Work}

VMcP is one the well research area in cloud computing [4, 18, 19, 20, 27, 28, 29, 30,31]. These research efforts can be classified into five categories based on their underlying techniques: heuristic bin packing $[18,19,20,33,21$, $22,23,24,25,12,26]$, biology-based optimization [27, 28, 29, 30, 31, 32], linear programming [33, 34], constraint programming [35], and simulated annealing optimization [36].

Heuristic bin packing. Many studies formulated the VM placement as vector bin packing, a well- known NP-hard optimization problem [19]. Simple heuristics like greedy algorithms are utilized to approximate the optimal solution of this NP-hard problem. These include worst fit and best fit in [18], first fit decreasing (FFD) and best fit decreasing (BFD) $[33,22]$. Improvement attempts in further research efforts resulted in an extended first fit decreasing (FFD) heuristics in the pMapper system [20,39], first fit and best fit modified using node utility and power consumption [21], worst fit based on the profiling data [23], first fit modified using application migration [24], best fit used for VM migration in [12], and a modified best fit decreasing heuristics in [26].

Biology-based Optimization. In [27], ant colony optimization method (ACO) is used to pack the VMs into the least number of physical machines necessary for the current workload. The SAPSO approach [28, 32] is a self-adaptive particle swarm optimization (PSO) algorithm. SAPSO has been applied to automatically adjust VM placement in response to changing resource pools in a dynamic cloud environment. The GABA approach [29] is a genetic algorithm (GA) based algorithm that dynamically reconfigures the VM mappings according to estimated future workload in a dynamic cloud environment.

Linear programming. In [33], server consolidation problem is considered as a bin packing, for which FFD and BFD are suggested. In addition, the server consolidation problem is formulated as a linear programming that is extended with a number of constraint types elicited from practical applications. An LP-relaxation-based heuristic is designed to minimize the cost of linear programming. The study in [34] formulates the QoS-aware VMcP problem as integer linear programing (ILP) and proposes a polynomial-time heuristic algorithm based on bipartite graph to reduce the complexity of ILP and efficiently solve the problem.

Constraint programming. A resource management framework is presented in [35]. It consists of two major components, a dynamic utility-based VM provisioning manager and a dynamic VM placement manager. Both managing tasks are modeled as constraint satisfaction problems. Entropy is a resource manager [40] used for homogeneous clusters, which employs constraint programing to perform dynamic consolidation and considers both VM placement and VM migration.

Simulated annealing optimization. The authors in [36] propose a dynamic runtime virtual machine mapping framework called GreenMap that contains multiple modules. The placement module utilizes a simulated annealing optimization based algorithm to dynamically map the VMs onto a small set of PMs that minimizes power consumption without significantly degrading the system performance.

Many VMcP schemes optimize a single objective such as resource utilization, power consumption or load balancing, etc. However, real-world VMcP solutions often need to consider multiple objectives. Take VMcP as an example, its optimization model has been widely studied and a number of characteristics have been taken into consideration, according to specific application scenarios, such as, VM dependency [41], inter-VM data movement $[41,42,43]$ and server load balance $[44,45,46,47]$. The characteristics considered in these research efforts have been formalized as objective functions or constraints. Meanwhile,recent research efforts tend to adopt evolutionary algorithm to address this kind multi-objective optimization problem. MGGA in [5] employs a grouping genetic algorithm with a fuzzy multi-objective evaluation to minimize the resource wastage, power consumption, and thermal dissipation costs. VMPACS in [31] is a multi-objective ant colony system algorithm (ACO) that minimizes both total resource wastage and power consumption. The work in [37] optimizes CPU utilization, network throughput, and disk I/O rate using a hybrid genetic algorithm for VMcP. This paper firstly incorporates five factors into the optimization model, which is more complex than the ones proposed in above research.

\section{Problem Formulation}

The first part of this section describes a universal resource wastage model, which support multiple resource dimensions. And then, a power consumption model has been built based on literatures review and experiments. Finally we formalize VMcP optimization problem. 


\subsection{Resource Wastage Model}

Different VMcP solutions may leave different amount of residual resources for each resource type on each PM. To accommodate future requests, an effective VMcP solution should keep the residual resources balanced in each dimension. Fig. 2 illustrated the problem of balancing residual resources. The cube represents the total CPU, memory and bandwidth capacity of a server. The two small cubes labelled VM1 and VM2 represent the amount of CPU, memory and bandwidth allocated to the two VMs. The left small cube labelled Residual Capacity denotes the remaining amount of CPU, memory and bandwidth after placing two VMs. Clearly, there is a lot of memory and bandwidth left but little CPU remaining. This CPU scarcity may block the placement of a new VM on this server.

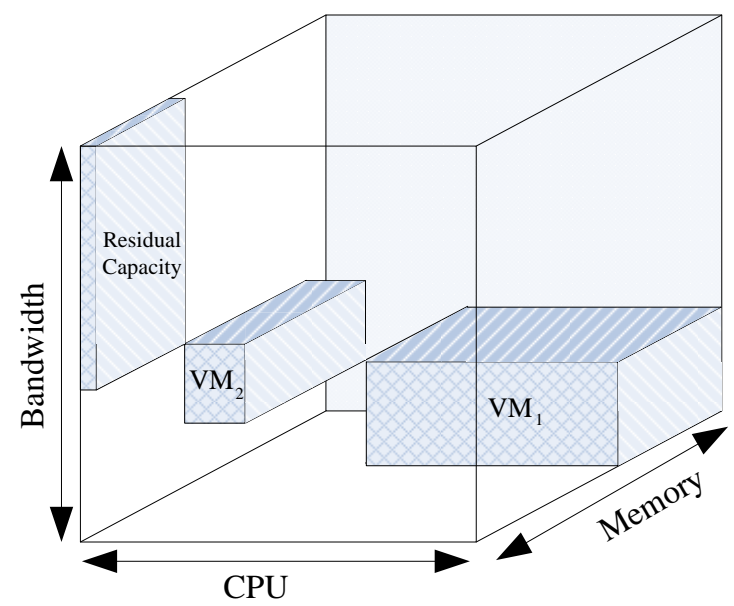

Fig. 2: An example of resources allocated to two VMs placed into a single PM

Based on the above observation, we propose resourceWastage $(j)$ function to quantify the cost of resource wastage in multiple loads on a server, which is an extension of the model in [30,31] used for the compatibility of multidimensional resource. The utilization of a resource (CPU, memory or network bandwidth) owned by a server is estimated as total amount that is requested by all VMs running on that server. $100 \%$ utilization of a specific resource may lead to a severe performance degradation [48], and may trigger live VM migration, which requires additional CPU time of the migrating node. Therefore, an upper bound is imposed on the resource utilization of a single server.

$$
\operatorname{resourceWastage}(j)=\frac{y_{j}}{R} \times \sum_{\phi \neq \rho} \sum_{\rho=1}^{R} \frac{\left|\left(T_{j}^{\phi}-\sum_{i=1}^{N}\left(\left(x_{i} \mid j\right) \cdot\left(j \mid x_{i}\right) \cdot D_{i}^{\phi}\right)\right)-\left(T_{j}^{\rho}-\sum_{i=1}^{N}\left(\left(x_{i} \mid j\right) \cdot\left(j \mid x_{i}\right) \cdot D_{i}^{\rho}\right)\right)\right|+\varepsilon}{\sum_{i=1}^{N}\left(\left(x_{i} \mid j\right) \cdot\left(j \mid x_{i}\right) \cdot D_{i}^{\phi}\right)+\sum_{i=1}^{N}\left(\left(x_{i} \mid j\right) \cdot\left(j \mid x_{i}\right) \cdot D_{i}^{\rho}\right)},
$$

where $D_{i}^{\phi}$ and $D_{i}^{\rho}$ are demands of resource $\phi$ and $\rho$ in VMi respectively. Let $T_{j}^{\phi}$ or $T_{j}^{\rho}$ be the threshold of the utilization of resource $\phi$ and $\rho$ in $j$-th server respectively. $R$ is the number of resource dimension and $\varepsilon$ is a tiny positive real number, which is 0.0001 in our experiments. We use an integer variable $x_{i}$ and a binary variable $y_{j}$. The value of variable $x_{i}$ indicates $x_{i}$-th server for $i$-th VM and the binary variable $y_{j}$ indicates whether $j$-th server is used (value 1 ) or not (value 0 ).

\subsection{Power Consumption Model}

A popular power consumption model $[49,50,51]$ shows that the power consumption is linearly proportional to the CPU utilization. We also investigate this model in Vmware ESXi 5.5 [52] deployed on an IBM x3850 X5 server. The performance data is collected by Veeam Monitor [53] every 2 hours from 10/2/2014 to 1/2/2015. The relationship between resource usages and power consumption is presented in Fig. 3. The correlation coefficient between them is listed in Table 1. It is obvious that the strong positive correlation exists between power consumption and CPU usage. 

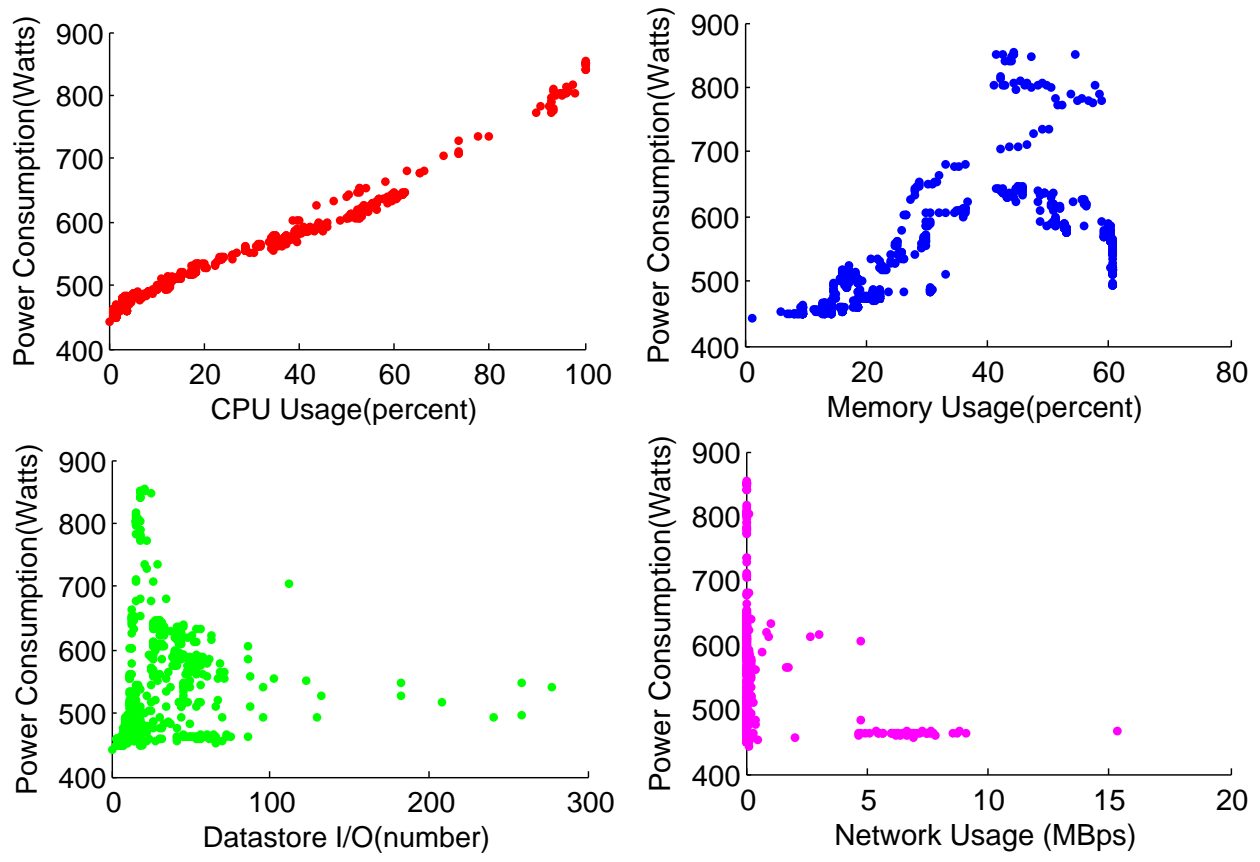

Fig. 3: Relationship between resource usages and power consumption

Table 1: Correlation coefficient between power consumption and resource usages

\begin{tabular}{lcc}
\hline Variable a & Variable b & Correlation Coefficient(a,b) \\
\hline \multirow{3}{*}{ Power Consumption } & CPU Usage & 0.990667 \\
\cline { 2 - 3 } & Memory Usage & 0.681875 \\
\cline { 2 - 3 } & Datastore I/O & 0.253151 \\
\cline { 2 - 3 } & Network Usage & -0.12116 \\
\hline
\end{tabular}

Based on above investigation, powerConsumption $(j)$ is introduced to quantify total power consumption by $j$-th server .

$$
\text { powerConsumption }(j)=y_{j} \times\left(\left(P_{j}^{\text {busy }}-P_{j}^{\text {idle }}\right) \cdot \sum_{i=1}^{N}\left(\left(x_{i} \mid j\right) \cdot\left(j \mid x_{i}\right) \cdot D_{i}^{C P U}\right)+P_{j}^{\text {idle }}\right),
$$

where $P_{j}^{\text {idle }}$ and $P_{j}^{\text {busy }}$ represent the power consumed when $j$-th server is idle and on full-load respectively. An idle server is turned off and it does not consume any power. $D_{i}^{C P U}$ refers to CPU utilization of $j$-th server .

\subsection{VMcP Formulation}

The VMcP optimization problem is described as follows: Suppose that there are $N$ VMs which are to be placed on $M$ physical machines, and none of the VMs requires more resource than a single server can offer. The resource allocation requests for a VM and the resource capacity of a PM (server) are both represented by a multi-dimensional vector. Each dimension refers to the amount of a specific type of resource requested by a VM or owned by a server. The aim is to simultaneously minimize power consumption and resource wastage.

Goal:

$$
\text { Minimize : } \sum_{j=1}^{M} \text { resourceWastage }(j)
$$




$$
\text { Minimize : } \sum_{j=1}^{M} \text { powerConsumption }(j)
$$

Constraints:

$$
\begin{gathered}
x_{i} \in\{1, \ldots, M\} \quad i=[1, \ldots, N] \\
\sum_{i=1}^{N} D_{i}^{r} \cdot\left(x_{i} \mid j\right) \cdot\left(j \mid x_{i}\right) \leq T_{j}^{r} \cdot y_{j} \quad j=[1, \ldots, M] \quad r=[1, \ldots, R] \\
y_{i}=\left\{\begin{array}{ll}
1 & \exists x_{i}=j \\
0 & \text { others }
\end{array} j=[1, \ldots, M]\right.
\end{gathered}
$$

The objectives in Eqs. 3 and 4 shown above are to minimize the power consumption and total resource wastage by all the servers. Constraint in Eq.5 ensures that each VM is allocated to one, and only one, server. Constraints in Eq.6 guarantees allocated resources from each server to not exceed their capacity. Constraint in Eq.7 defines the range of the variable $y_{j}$.

\section{VMPMBBO - Biogeography-based Optimization for VM Consolidated Placement}

In this section, we introduce background knowledge of biogeography-based optimization (BBO), provide an overview of the proposed VMPMBBO algorithm, and also provide discussion on migration and mutation.

\subsection{Biogeography-based Optimization (BBO)}

BBO is a family of biogeography-based optimization methods and can guarantee convergence to the optimal solution given enough generations [54]. Biogeography studies the geographical distribution of species migration and extinction of existing species and rise of new species. A geographically isolated habitat is called an island. The habitability (suitability for biological residence) of an island is indicated by its habitat suitability index (HSI), which is determined by a number of independent variables called Suitability Index Variables (SIVs). Temperature and rainfall are some examples of SIV.

The higher the HSI of an island is, the more the species on the island, the lower its immigration rate, and the higher its emigration rate. Species may migrate from high HSI islands to low HSI islands. The arrival of new species may increase the HSI of an island by increasing the diversity of species on the island. If the HSI of an island is too low, existing species on the island may become extinct. Unexpected random events like natural disasters or the sudden immigration of species from neighboring islands may cause a dramatic change in HSI of an island.

Biogeography-based optimization (BBO) applies biogeography to solving discrete optimization problems. In the original $\mathrm{BBO}$ [38], the population of candidate solutions is an archipelago of islands. A candidate solution is an island. The goodness (or fitness) of a solution with respect to an objective function is measured by its HSI. A good solution is an island with a high HSI. A poor solution is an island with a low HSI. The decision variables are SIVs. A solution is represented by a vector of SIVs.

There are two key operators in BBO: migration and mutation. The migration is designed to probabilistically share SIVs between solutions, thus increasing the quality of low HSI solutions. The mutation is used to probabilistically replace SIVs in a solution by randomly generating new SIVs. The initial population of candidate solutions evolves repeatedly from generation to generation until a termination criterion is met. In each repetition, a migration followed by a mutation is performed. Migration is a distinguishing feature of BBO from other population-based optimization methods.

There have been many extensions to the original BBO since its publication in 2008. One of them is BBO/Complex [39]. The original $\mathrm{BBO}$ is designed for optimizing a single system with a single objective and without any constraints. It considers an ecosystem that is an archipelago of islands. $\mathrm{BBO} /$ Complex is created for optimizing a complex system with multiple objectives and multiple constraints. The complex system may be composed of multiple subsystems that may have different objectives and different constraints. The ecosystem considered in BBO/Complex consists of multiple archipelagos, each of which contains a number of islands. The migration in BBO is extended to two types: migration between islands within the same subsystem and migration between islands in different subsystems. 
The second extension is a ranking algorithm used in within-subsystem migration that considers both the islands' performances and the system constraints. The third extension is a partial distance strategy used in cross-subsystem migration for effective diversity control.

\subsection{The Ecosystem Model in VMPMBBO}

In VMPMBBO, the VMcP problem is treated as a complex system consisting of multiple subsystems. Each subsystem optimizes itself with respect to its own objectives and constraints. They also share information with each other so that the entire complex system is optimized. The system decomposition is based on optimization objectives. A subsystem may optimize either power consumption (the objective in Eq.4) or resource wastage (the objective in Eq.3). Two subsystems may have the same optimization objective. No subsystem should violate the three constraints mentioned in Eqs.5-7. A subsystem consists of a number of candidate solutions.

VMPMBBO solves VMcP problem by using BBO/Complex to optimize the VMcP complex system. Each subsystem is an archipelago. A candidate $\mathrm{VMcP}$ solution in a subsystem is an island in an archipelago. Each candidate solution consists of $\left\{x_{1}, x_{2}, \ldots, x_{i}, \ldots x_{N}\right\}$, where the value of variable $x_{i}$ indicates the server number assigned to $i$-th VM.

Let $E=\left\{A_{1}, A_{2}, \ldots, A_{H}\right\}$ denote an ecosystem of $H$ archipelagos. $A_{k}=\left\{I_{k 1}, I_{k 2}, \ldots, I_{k T} ; O_{k} ; C_{1}, C_{2}, C_{3}\right\}$ represents an arbitrary archipelago, which contains T islands, one objective, and three constraints. The objective $O_{k}$ is either the power consumption or the resource wastage. The three constraints $C_{1}, C_{2}, C_{3}$ correspond to Eqs.5-7. All islands in the same archipelago have same objective $O_{k}$ and same constraints $C_{1}, C_{2}$ and $C_{3}$.

Each island is defined by a vector of $N$ SIVs. The $k t$-th island in archipelago $A_{k}$ is denoted by $I_{k t}=\left[S I V^{k t 1}\right.$, $\left.S I V^{k t 2}, \ldots, S I V^{k t N}\right]$, where $k \in\{1,2, \ldots, H\}, t=1,2, \ldots, T$. Each SIV is an integer that refers to the index of a server (physical machine) hosting a VM. $S I V^{k t i}$ is the index of the server occupied by the $i$-th VM in $I_{k t}$. The HSI of an island $I_{k t}$ is denoted by $H S I_{k t}$, which is computed as the inverse of the island's objective function $O_{k}$.

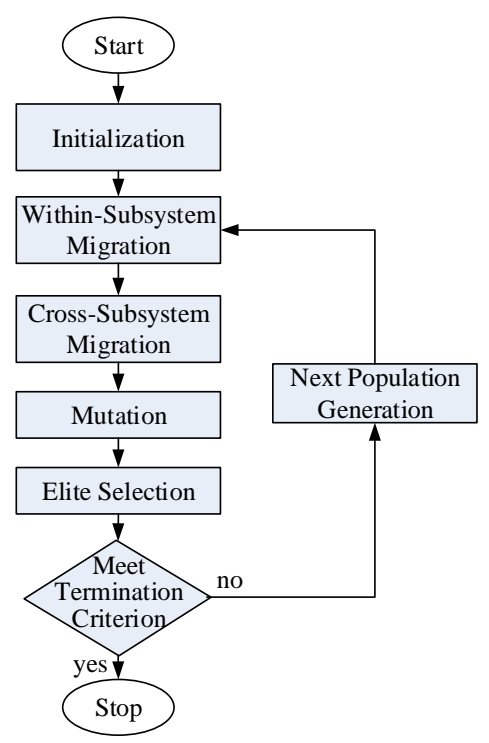

Fig. 4: VMPMBBO Flowchart

\subsection{VMPMBBO at a Glance}

The following are the major steps in VMPMBBO, as depicted in Fig. 4.

Step 1) Initialize the system

Step 2) Within-subsystem perform migration on each subsystem

Step 3) Perform cross-subsystem migration on selected subsystem pairs

Step 4) Perform probabilistic mutation on each island 
Step 5) Perform elitism 2).

Step 6) Terminate if the termination condition is satisfied otherwise, generate the next population and go to Step

In Step 1), the system parameters are initialized including both non-BBO parameters like maximum CPU and memory utilization, average power consumption by an idle or a busy server, and BBO parameters such as the number of subsystems(Subsystems), the number of islands(Popsize), the number of SIVs, stopping criterion, mutation $\operatorname{probability}\left(P_{\text {mutation }}\right)$, elitism parameter $\left(N^{\text {elite }}\right)$, and SIV migration probability $\left(P_{S I V m i g r a t i o n}\right)$. The initial population is number of subsystems multiplied by the number of islands per subsystem. Each subsystem is a matrix where each row is an island and each column is an SIV. Each SIV is randomly generated and it meets all three constraints corresponding to Eqs.5-7.

In Step 2), within-subsystem migration is based on the islands' within-subsystem ranking. In Step 3), crosssubsystem migration is based on similarity levels between subsystems. These two steps will be discussed in the following subsections in detail.

In Step 4), each island is mutated with mutation probability $P_{\text {mutation. }}$ If an island is chosen for mutation, we randomly select an SIV from this island and replace it with a new randomly-generated SIV.

In Step 5), a variant of modified non-dominated ranking system, as shown in algorithm 1 (for details refer to Section 4.4), is used to pick up $N^{\text {elite }}$ elitists from both optimal solutions of each subsystem and the elitists from last generation. And then, generate new population for next generation and replace $N_{\text {elite }}$ matrices with the elitists generated from last step, if the iteration doesn't reach the end. In Step 6), checking the termination criterion reaches the maximum number of (objective) function evaluations.

As shown in Fig. 5, there are $H$ subsystems (refer to $H$ archipelagos), $N^{\text {constraint }}$ constraints and $N^{\text {objective }}$ objective functions. Feasibility test and function evaluation are carried out on each subsystem, based on the constraints and objective functions that we have set. The feasible solutions of each objective have been obtained from different subsystems. The subsystems are loosely coupled and they communicate with each other by cross-subsystem migration. It provides an efficient way to communicate between subsystems and provides a unique migration strategy to share information both within and across subsystems. Cross-subsystem migration can significantly enrich communication among subsystems compared to more traditional methods [39].

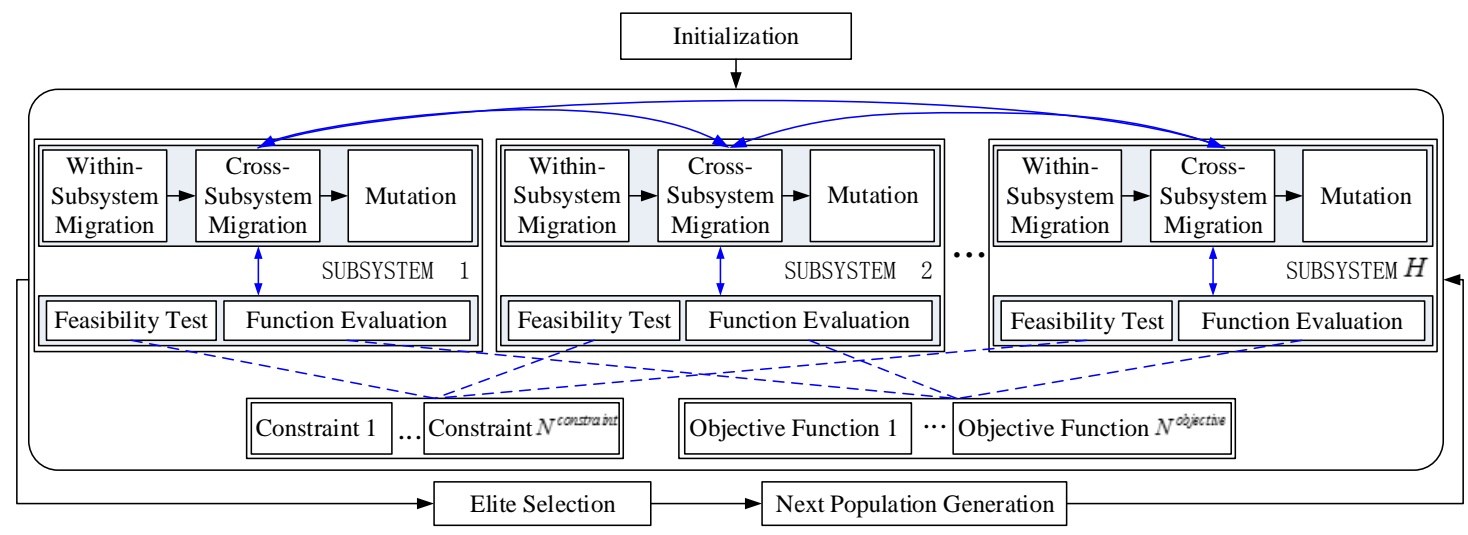

Fig. 5: Subsystems in VMPMBBO

\subsection{Within-Subsystem Migration in VMPMBBO}

Within-subsystem migration is executed in every subsystem based on the rankings of islands within their own subsystems. Firstly rank all islands in each subsystem by using a variation of ranking system in BBO/Complex. The rankings consider both the feasibility and performance of each island relative to other islands in the same subsystem. The feasibility is expressed in terms of number of constraint violations. The performance is expressed in terms of the objective function value. The feasibility is considered as a primary factor. The performance is secondary and has an impact only when two islands are equally feasible. The detailed algorithm is listed in Algorithm 1. Rank $k_{a}$ and Rank 
are the performance rank values of islands $I_{a}$ and $I_{b}$ respectively. A small performance rank value indicates a good solution.

Next, we probabilistically choose the immigrating islands based on the rankings. A low-ranking island (with a large performance rank value) has a higher probability of being chosen as an immigrating island than a high-ranking island (with a small performance rank value). The ranking $g$ of an island is converted to its immigration rate $\lambda$ using following formula:

$$
\lambda=\frac{\sum_{i=1}^{g} i / T}{\sum_{i=1}^{T} i / T},
$$

where $T$ is total number of islands in a subsystem. The probability of an island being chosen as an immigrating island is linearly related to its immigration rate.

Then, an emigrating island is probabilistically selected for each immigrating island using the roulette wheel selection [55] based on emigration rates. The emigration rate $\mu$ of an island is computed using the formula below. The larger the $\mu$ is, the higher the probability is.

$$
\mu=1-\lambda
$$

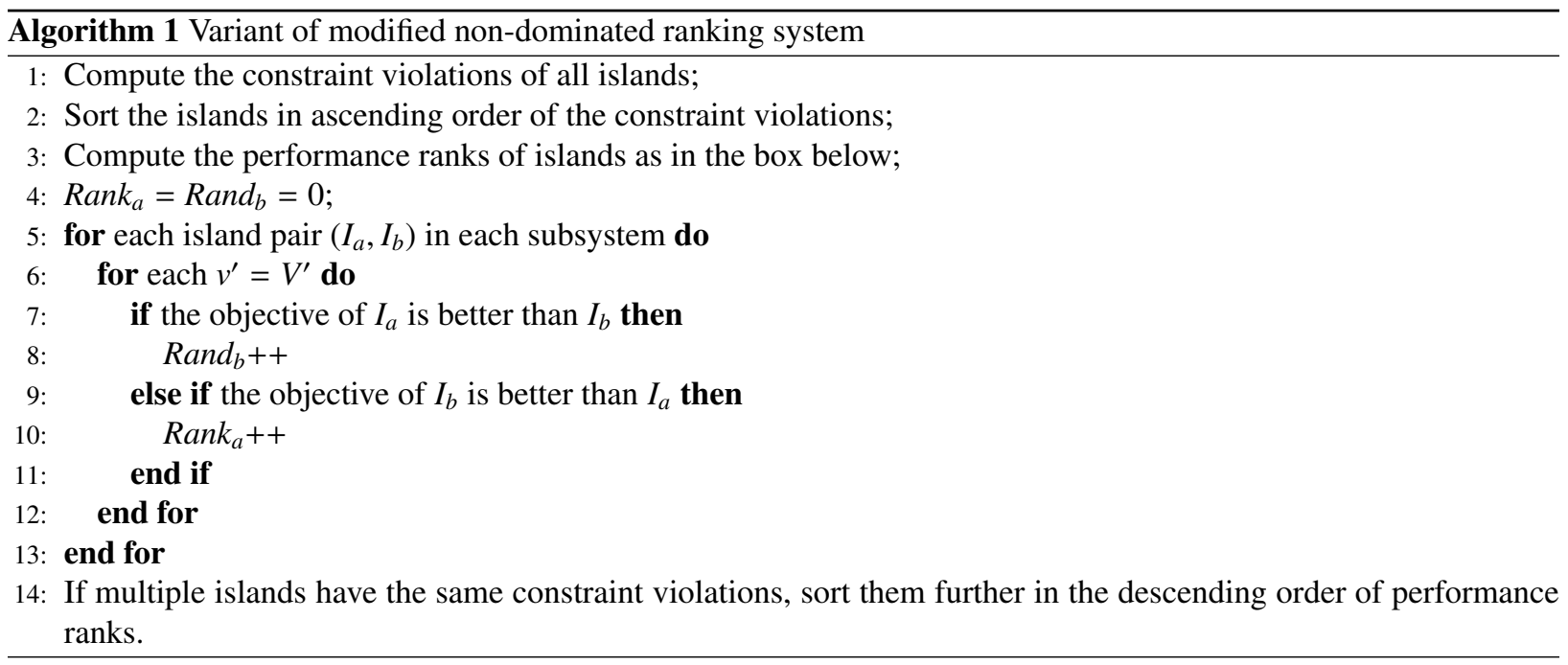

In last step perform migration from chosen emigrating island to corresponding immigrating island. Each SIV in the immigrating island has probability $P_{\text {SIVmigration }}$ of being replaced by a randomly selected SIV from the emigrating island.

\subsection{Cross-Subsystem Migration in VMPMBBO}

Cross-subsystem migration is carried out only on selected subsystem pairs. First, we compute the constraint similarity level (CSL) and objective similarity level (OSL) between every two subsystems. The detail is listed in Algorithm 2. It is based on fast similarity level calculation (FSLC) [39]. SL represents either CSL or OSL. V denotes the constraint set or objective set of one subsystem while $\mathrm{V}^{\prime}$ is the corresponding counterpart in other subsystem in a pair. In VMPMBBO, all subsystems have same three constraints (Eqs.5-7). Therefore the value of CSL is always 3 , and any two subsystems may have either the different objectives or the same objective. So the value of $O S L$ corresponds to 0 or 1 .

Next, compute the migration probability $P_{\text {migration }}$ based on subsystem similarity level according to Eq.10. $O S L_{\max }$ and $C S L_{\max }$ are the maximum OSL and CSL values in the population. In VMPMBBO, the computed $P_{\text {migration }}$ is 0.5 . 


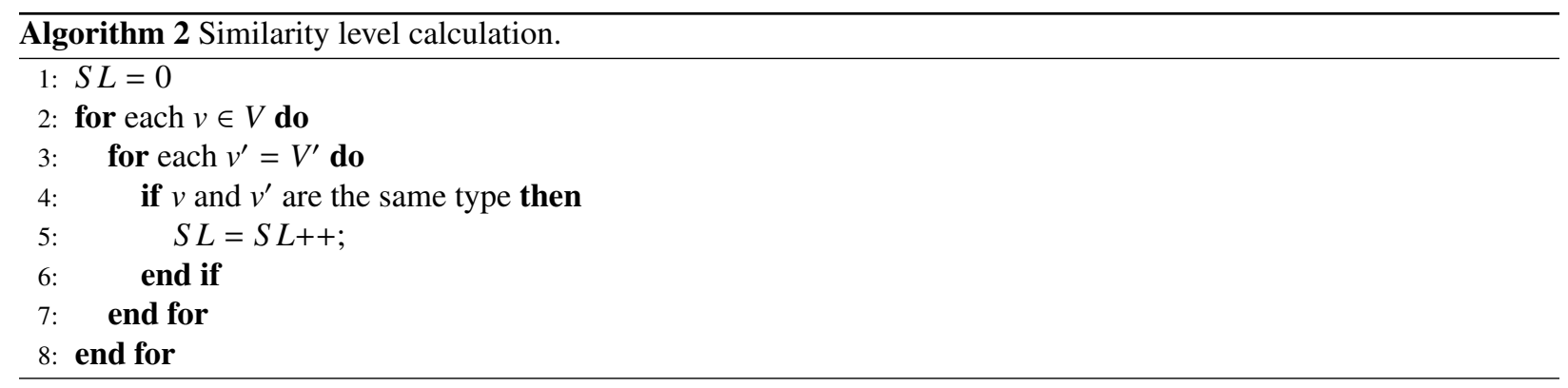

The probability of migration between two subsystems is linearly related to $P_{\text {migration }}$.

$$
P_{\text {migration }}= \begin{cases}\frac{1}{2}\left(\frac{O S L}{O S L_{\max }}+\frac{C S L}{C S L_{\max }}\right), & \text { if } O S L_{\max }>0 \text { and } C S L_{\max }>0 \\ \frac{1}{2} \frac{O S L_{\max }}{O S L_{\max }}, & \text { if } O S L_{\max }>0 \text { and } C S L_{\max }=0 \\ \frac{1}{2} \frac{C S L L_{\max }}{C S L_{\max }} & \text { if } O S L_{\max }=0 \text { and } C S L_{\max }>0 \\ 0, & \text { ifOS } L_{\max }=0 \text { and } C S L_{\max }=0\end{cases}
$$

Then, for each subsystem pair chosen for inter-subsystem migration, select immigrating islands from one subsystem with probability $P_{\text {interSysimg }}$. For each immigrating island, compute distances between this island and all islands in other subsystem. Then select an emigrating island using the roulette wheel algorithm based on these distances. The partial distance proposed in BBO/Complex is not used. Instead we used the Euclidean distances between island vectors because these vectors have same structure. Last step perform the migration between the chosen island pair in the chosen subsystem pair. Each SIV in immigrating island has probability $P_{S I V m i g r a t i o n}$ of being replaced by a randomly selected SIV from emigrating island

\subsection{Discussion}

The immigration rate $\lambda$ and emigration rate $\mu$ are important parameters in the configuration of VMPMBBO. Our chosen configuration, $\lambda$ as described in Eq.8 and $\mu$ in Eq.9, are essentially quadratic with respect to the ranking $g$ of each island (the HSI of an island), as shown in Fig. 6. $\lambda$ can be transformed to Eq.9 below, where $a$ and $b$ represent constants.

$$
\lambda=a(g / T)^{2}+b(g / T)
$$

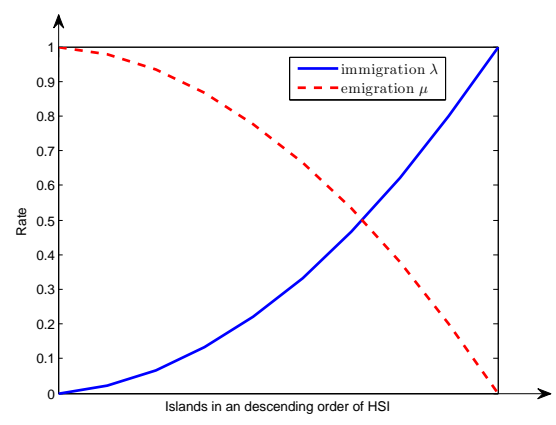

Fig. 6: Graph of immigration rate $\lambda$ and emigration rate $\mu$ in VMPMBBO

In the original BBO [38], a linear strategy described in Eq.12 is used. Quadratic in Eq.13, cosine in Eq.14, and exponential strategies in Eq.15 have been proposed in [56, 57] for improving performance. In next section we will present our experimental results of these strategies when they are applied to proposed VMPMBBO system. The 
quadratic strategy in Eq.11 performs the best in these experiments.

$$
\begin{aligned}
\lambda & =\frac{g}{T} \\
\lambda & =\left(\frac{g}{T}\right)^{2} \\
\lambda & =0.5\left(1-\cos \left(\frac{\pi}{T} \pi\right)\right) \\
\lambda & =e^{g / T}
\end{aligned}
$$

\section{Experiments and Analysis}

In this section, we evaluate the effectiveness of VMPMBBO applied to VMcP. For this purpose, a series of experiments have been carried out. First of all, we compare experimental results of two VMP processes with and without VMcP. Then we present the evaluation results comparing proposed VMPMBBO with two existing multiobjective VMcP algorithms, MGGA [30] and VMPACS [31]. The experimental results also verified the robustness of VMPMBBO. The parameter values concerning experiments have also been discussed. A total of 35 scenarios have been used, and both synthetic and real datasets have been used, as shown in Table 2.

Table 2: Scenarios and datasets

\begin{tabular}{lll}
\hline Evaluation & Scenario Index & Test Dataset \\
\hline Necessity Proving & $1-10$ & Synthetic datasets \\
Solution Quality & 11,12 & Real dataset -EC2 \\
& $13-22$ & Synthetic datasets \\
Computational Efficiency & 23 & Real dataset -EC2 \\
& 24,26 & Real dataset -XJTUDLC \\
& 27 & Synthetic datasets \\
Robustness & 28 & Real dataset -EC2 \\
Parameter Value Selection & $29,30,31$ & Real dataset -XJTUDLC \\
\hline
\end{tabular}

Simulation Configurations: VMPMBBO is configured using parameters shown in Table 3. The term "Subsystems" is the number of subsystems. The "popsize" refers to the number of islands per subsystem (archipelago). Subsystems and popsize are fixed as 4 and 3 respectively in most experiments with the exception of one scenario where the impact of these two parameters is tested. MGGA and VMPACS are configured as recommended in [30,31]. For each algorithm and each scenario, 10 Monte Carlo simulations are conducted, and the average results are used in the comparisons. According to complexity of the different datasets, the termination criterion (preset maximum number of function evaluations) is 100,000 for synthetic dataset and 10,000 for real datasets, respectively. The programs for proposed algorithm, MGGA and VMPACS were coded in the matlab 7.0 and ran them on a Virtual Machine with 4 vCPU and 8 GB RAM hosted on a VMWARE ESXi 5.5 of a Dell R720 server.

\subsection{Necessity of dealing with VMcP in VMP}

In this section, we prove the necessity of VMcP applied to VMP. For this purpose, we compare the experimental results of two VMP processes with and without VMcP. The process without VMcP is actually a single process with $\mathrm{VMiP}$, which deals with continuous arrival of $\mathrm{VM}$ deployment and removal requests at runtime. The process with $\mathrm{VMcP}$ is a mixed process comprise of VMiP and VMcP, where VMiP is used to deal with continuous VM requests and $\mathrm{VMcP}$ is used to consolidate existing VMs.

1) Dataset

The experiments are conducted using both the synthetic data from related literature [31] and the data from Amazon EC2 [58]. 
Table 3: Parameter setup for experiments

\begin{tabular}{cc}
\hline Parameter & Value \\
\hline Subsystems & 4 \\
Popsize & 3 \\
$P_{\text {SIVmigration }}$ & 0.5 \\
$P_{\text {mutation }}$ & 0.05 \\
$P_{\text {interSysImg }}$ & 0.5 \\
$N^{\text {elite }}$ & 1 \\
\hline
\end{tabular}

Synthetic Dataset. The same configuration of synthetic data as mentioned in [31] is adopted for the purpose of performance evaluation with following considerations:

1. In the solution procedure of VMcP problem, the number of candidate servers is set as the number of VMs in order to incorporate the worst VM placement scenario (one VM per server). It is obvious that reducing the number of optional servers will narrow the search space and improve efficiency.

2. The servers are assumed to be homogeneous. $P_{j}^{\text {idle }}$ and $P_{j}^{\text {busy }}$ in Eq.2 are set to 162 and 215 Watt respectively. The CPU and memory utilizations of each server is capped at $90 \%\left(T_{j}^{C P U}=T_{j}^{M E M}=90 \%\right)$. However, VMPMBBO is also applicable in the case of heterogeneous servers.

3. Each VM deployment request is a pair of CPU and memory demands. It is assumed that CPU and memory demands are linearly correlated. All VM deployment requests are randomly generated using the method presented in [59] listed in Algorithm 3. $\xi_{c}, \xi_{m}$, and $\gamma$ are all random numbers between 0 and 1. $\overline{D^{C P U}}$ and $\overline{D^{M E M}}$ are reference values of CPU and memory demands, respectively. $D_{i}^{C P U}$ and $D_{i}^{M E M}$ together form a deployment request. $\rho$ is a probability value used to control the correlations of CPU and memory demands.

Based on above settings, we generated VM deployment requests in 10 different scenarios by changing $\overline{D^{C P U}}$ and $\overline{D^{M E M}}$ twice $(25 \%, 45 \%)$, and $P$ five times $(0.0,0.25,0.50,0.75,1.0)$. The ranges of the generated CPU and memory demands are $[0,50 \%)$ with $\overline{D^{C P U}}$ and $\overline{D^{M E M}}$ being $25 \%$, and $[0,90 \%)$ with $\overline{D^{C P U}}$ and $\overline{D^{M E M}}$ being $45 \%$. The average correlation coefficients of CPU and memory demands are $-0.754,-0.348,-0.072,0.371$, and 0.755 per request set to $\overline{D^{C P U}}$ and $\overline{D^{M E M}}$ being $25 \%$. The five coefficients indicate strong negative, weak negative, no, weak positive, and positive correlations in sequence. The coefficients are $-0.755,-0.374,-0.052,0.398$, and 0.751 when $\overline{D^{C P U}}$ and $\overline{D^{M E M}}$ are $45 \%$. The CPU and memory utilizations of each server are both capped at $90 \%\left(T_{j}^{C P U}=T_{j}^{M E M}=90 \%\right)$ in all experiments.

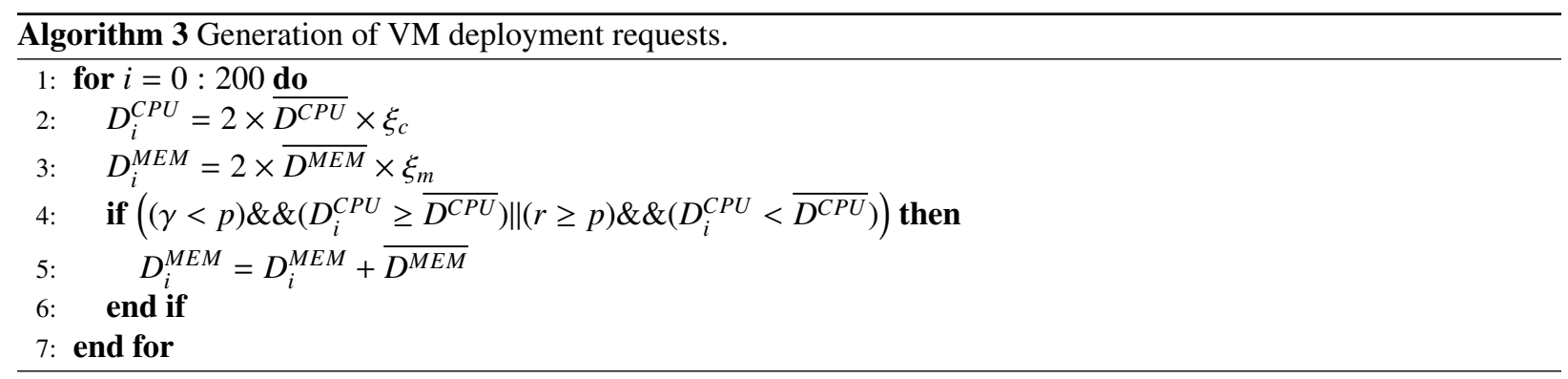

Real Dataset-EC2 We consider the resource requirement relevant to the types of VM instances provided by Amazon EC2 [58]. It is a public cloud. We take 17 instance types into account in our simulation, including 7 general instances, 5 compute optimized instances, and 5 memory optimized instances. The details are shown in Table 4 . It is obvious that the CPU demand and memory demand of instances has strong positive correlation. The CPU models have been presented in [58]. The number of CPUs and the size of memory in each server is assumed as mentioned in Table 4. As E5-2680 v2 has 10 physical cores. And with Hyper-Threading (HT) technology, each E5-2680 v2 can 
Table 4: VM and PM configurations in real dataset-EC2

\begin{tabular}{|c|c|c|c|c|c|}
\hline \multirow{2}{*}{ Pattern } & \multicolumn{3}{|c|}{ Instance Specs } & \multicolumn{2}{|c|}{ Server Specs } \\
\hline & Instance Type & vCPU & Memory (GiB) & $\mathrm{CPU}$ & Memory(GiB) \\
\hline \multirow{8}{*}{$\begin{array}{l}\text { General } \\
\text { Purpose }\end{array}$} & t2.micro & 1 & 1 & \multirow{3}{*}{$\begin{array}{l}2 * \text { Intel E5-2620 v2 } \\
24 \text { logic processors }\end{array}$} & \multirow{3}{*}{32} \\
\hline & t2.small & 1 & 2 & & \\
\hline & t2.medium & 2 & 4 & & \\
\hline & m3.medium & 1 & 3.75 & & \multirow{5}{*}{128} \\
\hline & m3.large & 2 & 7.5 & $2 *$ Intel E5-2670 v2 & \\
\hline & $\mathrm{m} 3$ xlarge & 4 & 15 & 40 logic processors & \\
\hline & $\mathrm{m} 3.2 x$ large & 8 & 30 & & \\
\hline & c3.large & 2 & 3.75 & & \\
\hline \multirow{4}{*}{$\begin{array}{l}\text { Compute- } \\
\text { Optimized }\end{array}$} & c3.xlarge & 4 & 7.5 & & \multirow{4}{*}{128} \\
\hline & c3.2xlarge & 8 & 15 & $4 *$ Intel E5-2680 v2 & \\
\hline & c3.4xlarge & 16 & 30 & 80 logic processors & \\
\hline & c3.8xlarge & 32 & 60 & & \\
\hline \multirow{5}{*}{$\begin{array}{l}\text { Memory- } \\
\text { Optimized }\end{array}$} & r3.large & 2 & 15.25 & & \multirow{5}{*}{512} \\
\hline & r3.xlarge & 4 & 30.5 & & \\
\hline & r3.2xlarge & 8 & 61 & 4*Intel E5-2670 v2 & \\
\hline & r3.4xlarge & 16 & 122 & 80 logic processors & \\
\hline & r3.8xlarge & 32 & 244 & & \\
\hline
\end{tabular}

provide 20 logical cores, that is each E5-2680 can serve 20 vCPU [58]. Maximum number of vCPU demand of a memory optimized instance is 32, and each server is assumed to have four CPU (E5-2680), and provide 80 logical processors. If using five memory-optimized instance types, the demand of memory is 7.625 times larger than that of vCPU in quantitative terms. Therefore, the memory of each server is assumed to be $512 \mathrm{GiB}$, which is the power of 2 and is close to $610(80 * 7.625)$.

Based on above configuration, the deployment requests of each instance type independently follow uniform distributions, which has been widely adopted in previous researches $[10,60] . P_{j}^{i d l e}$ of the 4 server models are set to 110 , 150, 250, 200 Watt respectively. And $P_{j}^{\text {busy }}$ are set to 300, 350, 700, 600 Watt respectively.

2) Result Discussion

The first 10 scenarios (Scenarios 1-10 in Table 2) are based on Synthetic Dataset. In each scenario, we generate a sequence of $400 \mathrm{VM}$ requests to simulate the continuous arrival requests. It is assumed that $75 \%$ of the VM requests are deployment requests, and the others are removal requests. The removal of only deployed VM is guaranteed. A best fit algorithm (Algorithm 4) is adopted for each VM request, and both resource wastage and power consumption are considered. In the process with $\mathrm{VMcP}, \mathrm{VMcP}$ started after $400 \mathrm{VM}$ requests and is used to consolidate the existing VMs (200 VMs).

Table 5 lists the experiment results of total resource wastage and power consumption with and without VMcP. It is obvious that VMPMBBO plays an important role in VMP.

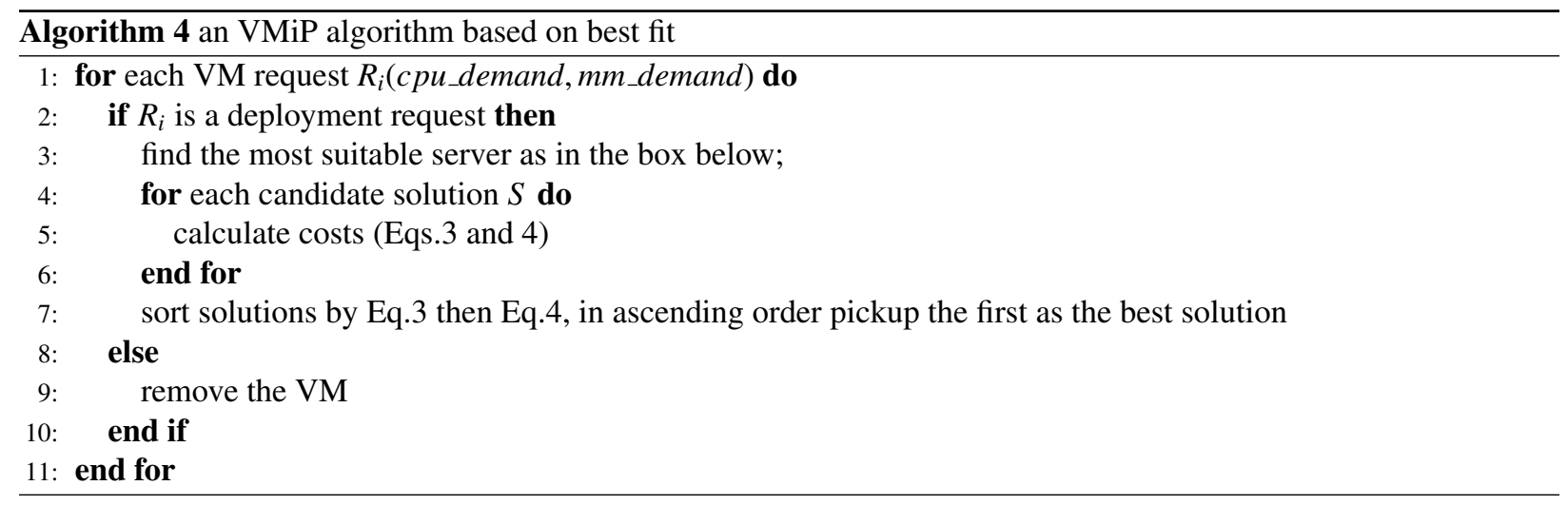


Table 5: Power consumption and resource wastage of best fit and VMPMBBO algorithms.

\begin{tabular}{|c|c|c|c|c|}
\hline Reference value & Corr. & Algorithm & Power consumption(W) & Resource wastage \\
\hline \multirow{14}{*}{$\overline{D^{C P U}}=\overline{D^{M E M}}=25 \%$} & \multirow{2}{*}{-0.754} & Best Fit & 13161.2361 & 4.4348 \\
\hline & & VMPMBBO & 11913.23607 & 1.20919 \\
\hline & \multirow{2}{*}{-0.348} & Best Fit & 11790.7176 & 4.2429 \\
\hline & & VMPMBBO & 11304.71756 & 1.17909 \\
\hline & \multirow{2}{*}{-0.072} & Best Fit & 11228.8508 & 3.5225 \\
\hline & & VMPMBBO & 10904.85084 & 0.92431 \\
\hline & \multirow{2}{*}{0.371} & Best Fit & 11229.2016 & 3.1733 \\
\hline & & VMPMBBO & 10590.78346 & 0.73268 \\
\hline & \multirow[b]{2}{*}{0.755} & Best Fit & 10645.0821 & 2.18512 \\
\hline & & VMPMBBO & 10358.42339 & 0.54919 \\
\hline & \multirow{2}{*}{-0.755} & Best Fit & 24117.3295 & 21.1098 \\
\hline & & VMPMBBO & 22380.15586 & 9.80711 \\
\hline & \multirow{2}{*}{-0.374} & Best Fit & 23565.6729 & 19.797 \\
\hline & & VMPMBBO & 21297.67288 & 6.53505 \\
\hline \multirow{6}{*}{$\overline{D^{C P U}}=\overline{D^{M E M}}=45 \%$} & \multirow{2}{*}{-0.052} & Best Fit & 22428.1293 & 13.7283 \\
\hline & & VMPMBBO & 20646.12933 & 5.5952 \\
\hline & \multirow{2}{*}{0.398} & Best Fit & 21659.5739 & 13.7113 \\
\hline & & VMPMBBO & 19890.57741 & 5.54417 \\
\hline & \multirow{2}{*}{0.751} & Best Fit & 19839.6558 & 6.62257 \\
\hline & & VMPMBBO & 18770.45581 & 2.59881 \\
\hline
\end{tabular}

The last 2 scenarios (Scenario 11, Scenario 12) are based on Real Dataset-EC2.

Scenario 11 consists of 5 phases: VMiP I, VMcP I, VMiP II, VMcP II and VMiP III.

The request sequence presents continuously deployment and removal of VMs over time. The VM requests in each sequence have been randomly generated on Real Dataset-EC2. VMiP I simulates the phase at the system startup, when there is no prior placement. In VMiP I, a request sequence of 5,000 VM requests (Sequence I) has been simulated, including 1,000 removal requests. In VMcP I, VMPMBBO is used to consolidate the residual 4,000 VMs after VMiP I. In VMiP II, a new sequence of 6,000 VM requests (Sequence II) has been simulated, including 2,000 removal requests. The residual 8,000 VMs have been consolidated in VMcP II. After that, a sequence of 6,000 VM requests (Sequence III) is processed in VMiP III, including 2,000 removal requests.

Scenario 12 simulates a single process with VMiP and the request sequence is a combination of Sequence I, II and III.

Table 6: Power consumption and resource wastage in Scenarios 11 and 12

\begin{tabular}{ccccccc}
\hline & \multicolumn{3}{c}{ Power consumption(W) } & \multicolumn{3}{c}{ Resource wastage } \\
& $\begin{array}{c}\text { Scenario } \\
12\end{array}$ & $\begin{array}{c}\text { Scenario } \\
11\end{array}$ & $\begin{array}{c}\text { Decline } \\
\text { percentage }\end{array}$ & $\begin{array}{c}\text { Scenario } \\
\text { Scenario } \\
12\end{array}$ & $\begin{array}{c}\text { Decline } \\
\text { percentage }\end{array}$ \\
\hline $\begin{array}{c}\text { When the 5,001th request } \\
\text { coming }\end{array}$ & 229135 & 205112 & $10.484 \%$ & 49.2377 & 43.8525 & $10.9371 \%$ \\
$\begin{array}{c}\text { When the 11,001th request } \\
\text { coming }\end{array}$ & 413175 & 366876 & $11.206 \%$ & 87.9862 & 76.0912 & $13.5192 \%$ \\
\hline
\end{tabular}

Fig. 7(a) and Fig. 7(b) show the costs while VM request arriving with (Scenario 11) or without (Scenario 12) VMPMBBO, respectively. The solid curves in Fig. 7 show the variations of total power consumption and total resource wastage at each request respectively. The reduction of costs for some requests is a result of applying VMPMBBO. As can been seen from Table 6, total power consumption has been reduced by more than 10 percent by adopting VMPMBBO.

\subsection{Solution Quality}

In this section, the performance of the three algorithms, MGGA, VMPACS and VMPMBBO, is compared and contrasted. 


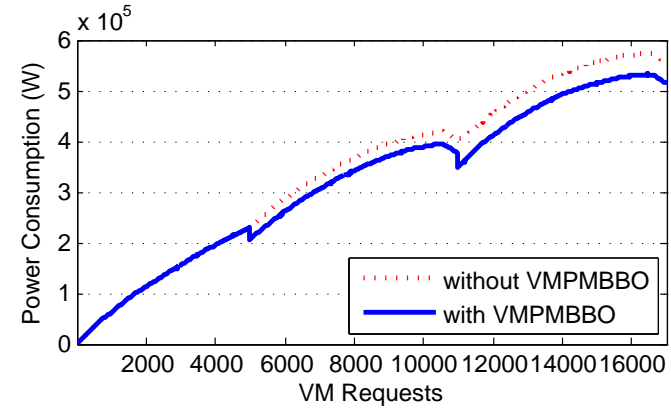

(a) Total power consumption curves

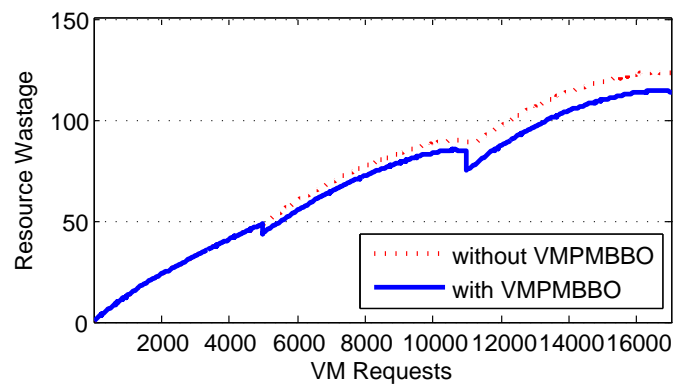

(b) Total resource wastage curves

Fig. 7: Mean cost curves with and without VMPMBBO

\section{1) Dataset}

Three kinds of datasets have been used in Scenarios 13-24.

Scenarios 13-22 are based on 10 synthetic datasets, which have been used in VMcP as described in Section 5.1. Each synthetic dataset contains $200 \mathrm{VMs}$.

The Scenario 23 is based on 8,000 VMs consolidated in VMcP II as shown in Section 5.1.

Real Dataset-XJTUDLC. In Scenario 24, we have collected data from a real-world data center, which is a private cloud powered by VMware ESXi 5.5. This data center belongs to the College of Distant Learning of Xi'an Jiaotong University,China (XJTUDLC for short, www.dlc.xjtu.edu.cn), which serves more than 81,000 e-Learning students. The details of VM instances in data center are shown in Table 7. Moreover, the physical servers of the datacenter are listed in Table 8.

Table 7: VM instances in the data center

\begin{tabular}{lllllllll}
\hline Instance Type & 1 & 2 & 3 & 4 & 5 & 6 & 7 & 8 \\
\hline vCPU & 1 & 2 & 2 & 2 & 4 & 4 & 8 & 16 \\
Memory (GiB) & 2 & 4 & 8 & 16 & 8 & 16 & 16 & 8 \\
VM Quantity & 9 & 48 & 17 & 11 & 36 & 16 & 10 & 4 \\
\hline
\end{tabular}

Table 8: Server hardware configuration in the data center of XJTUDLC

\begin{tabular}{cccccc}
\hline Server Model & Logic Processor & Memory $(\mathrm{GiB})$ & $P_{j}^{\text {idle }}(W)$ & $P_{j}^{\text {busy }}(W)$ & quantity \\
\hline DELL R720 & $\begin{array}{c}2 * \text { Intel E5-2650 } \\
32 \text { logic processors } \\
4 * \text { Intel E7-4850 } \\
\text { IBM X3850 X5 }\end{array}$ & 64 & 123 & 317 & 22 \\
& 80 logic processors & 256 & 443 & 854 & 3 \\
\hline
\end{tabular}

\section{2) Result Evaluation}

The comparison of results in Scenarios 13-22 are shown in Table 9 and Fig. 8 to Fig. 17. Table 9 lists the total resource wastage and power consumption of each algorithm after 100,000 function evaluations. We use mean cost curve to depict convergence of each algorithm as shown in Figs. 8-17. Each point on the curve represents the mean value of cost calculated from each function evaluation. And we use boxplot to depict statistical distribution of cost values through their quartiles. These cost values are calculated from each function evaluation. The median in boxplot refers to the middle value of costs, which has been calculated from a total of 100,000 function evaluations for synthetic dataset and 10,000 for real datasets.

Fig. 10(a) and Fig. 10(b) plot the mean cost curve of each objective function in all three algorithms when $\overline{D^{C P U}}=\overline{D^{M E M}}=25 \%$, Corr. $=-0.072$. Both show that VMPMBBO is superior to MGGA and VMPACS in this scenario. VMPMBBO can converge to a lower minimum faster than MGGA and VMPACS. Fig. 10(c) and Fig. 10(d) 
Table 9: Power consumption and resource wastage of VMPMBBO and other two multi-objective algorithms.

\begin{tabular}{|c|c|c|c|c|}
\hline Reference value & Corr. & Algorithm & Power consumption & Resource wastage \\
\hline \multirow{15}{*}{$\overline{D^{C P U}}=\overline{D^{M E M}}=25 \%$} & \multirow[t]{3}{*}{-0.754} & MGGA & 12075.23607 & 2.87269 \\
\hline & & VMPACS & 12075.23607 & 1.94735 \\
\hline & & VMPMBBO & 11913.23607 & 1.20919 \\
\hline & \multirow[t]{3}{*}{-0.348} & MGGA & 11466.71756 & 1.89013 \\
\hline & & VMPACS & 11466.71756 & 1.74654 \\
\hline & & VMPMBBO & 11304.71756 & 1.17909 \\
\hline & \multirow[t]{3}{*}{-0.072} & MGGA & 11066.85084 & 2.40885 \\
\hline & & VMPACS & 10904.85084 & 1.37153 \\
\hline & & VMPMBBO & 10904.85084 & 0.92431 \\
\hline & \multirow[t]{3}{*}{0.371} & MGGA & 10914.78346 & 1.65359 \\
\hline & & VMPACS & 10914.78346 & 1.16171 \\
\hline & & VMPMBBO & 10590.78346 & 0.73268 \\
\hline & \multirow[t]{3}{*}{0.755} & MGGA & 10520.42339 & 1.25547 \\
\hline & & VMPACS & 10520.42339 & 1.00769 \\
\hline & & VMPMBBO & 10358.42339 & 0.54919 \\
\hline \multirow{15}{*}{$\overline{D^{C P U}}=\overline{D^{M E M}}=45 \%$} & \multirow[t]{3}{*}{-0.755} & MGGA & 22704.15586 & 13.0427 \\
\hline & & VMPACS & 22380.15586 & 11.5713 \\
\hline & & VMPMBBO & 22380.15586 & 9.80711 \\
\hline & \multirow[t]{3}{*}{-0.374} & MGGA & 21783.67288 & 9.75471 \\
\hline & & VMPACS & 21621.67288 & 8.11456 \\
\hline & & VMPMBBO & 21297.67288 & 6.53505 \\
\hline & \multirow[t]{3}{*}{-0.052} & MGGA & 21294.12933 & 10.8148 \\
\hline & & VMPACS & 20970.12933 & 7.59448 \\
\hline & & VMPMBBO & 20646.12933 & 5.5952 \\
\hline & \multirow[t]{3}{*}{0.398} & MGGA & 20214.57741 & 7.28013 \\
\hline & & VMPACS & 20052.57741 & 6.23694 \\
\hline & & VMPMBBO & 19890.57741 & 5.54417 \\
\hline & \multirow[t]{3}{*}{0.751} & MGGA & 18770.45581 & 3.56561 \\
\hline & & VMPACS & 18932.45581 & 3.02583 \\
\hline & & VMPMBBO & 18770.45581 & 2.59881 \\
\hline
\end{tabular}

boxplots the statistical distribution of each objective function values in the same scenario. It is revealed that the upper quartile value of VMPMBBO is smaller than the minimum values of the other two algorithms. In another word, more than $75 \%$ objective function values of VMPMBBO are lower (i.e. better) than the smallest objective values (best results) of MGGA and VMPACS. Similar results are obtained in other four scenarios when $\overline{D^{C P U}}=\overline{D^{M E M}}=25 \%$. These figures are portrayed in Fig. 9 to Fig. 12.

The cost curves when $\overline{D^{C P U}}=\overline{D^{M E M}}=45 \%$, Corr. $=-0.755$ are plotted in Fig. 13(a) and Fig. 13(b). The initial performance of VMPMBBO is better than MGGA and worse than VMPACS. However, VMPBBO can guarantee convergence to better solutions given enough generations. The boxplots in Fig. 13(c) and Fig. 13(d) are similar to shown in Fig. 10(c) and Fig. 10(d). They also support similar statistical performance of VMPMBBO in this scenario. In the other four correlation scenarios when $\overline{D^{C P U}}$ and $\overline{D^{M E M}}$ are $45 \%$, VMPMBBO also performs similarly. The figures are included in Fig. 14 to Fig. 17.

In summary, VMPMBBO achieved even better in most cases or competitive performance, compared to MGGA and VMPACS. VMPMBBO excels when most VM requests are not demanding.

The Scenarios 23 and 24 are based on Real Dataset-EC2 and Real Dataset-XJTUDLC respectively.

The results on two Real Datasets have been presented in Figs.18 and 19, where the termination criterion is 10,000 function evaluations.

We have also carried out experiments with 100,000 function evaluations. The results have been presented in Figs.20 and 21. However, we find that the solution after 10,000 function evaluations is acceptable.

We can conclude from experimental results that VMPMBBO has superior in most cases or at least competitive performance compared to MGGA and VMPACS in both synthetic and real datasets. 


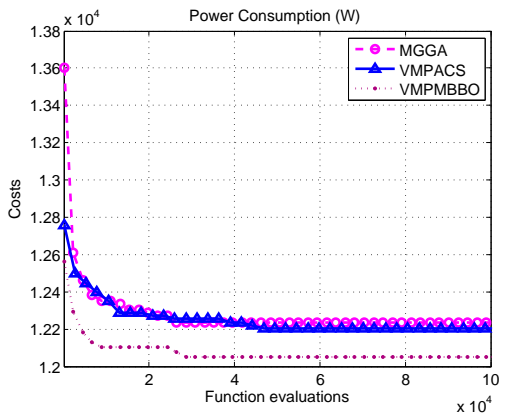

(a)

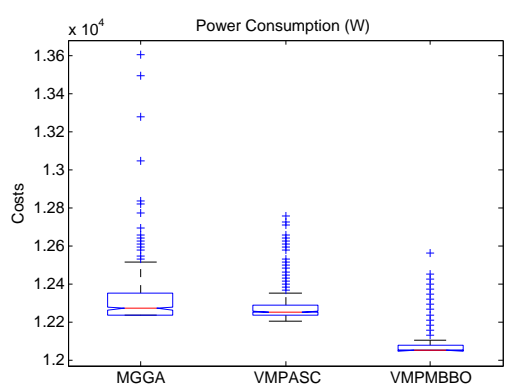

(c)

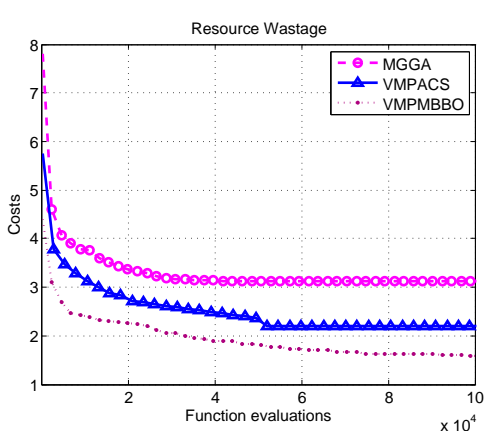

(b)

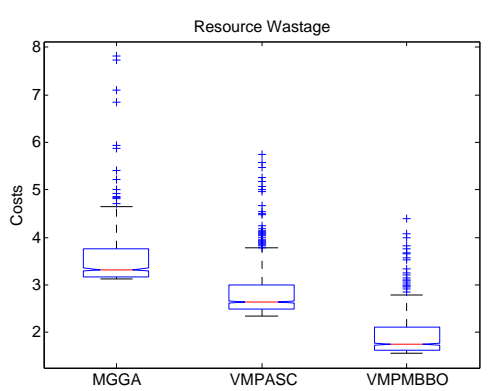

(d)

Fig. 8: Mean cost curve and boxplot of each objective in $\overline{D^{C P U}}=\overline{D^{M E M}}=25 \%$, Corr. $=-0.754$

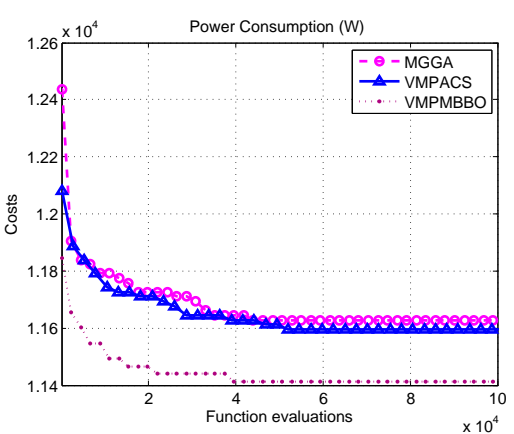

(a)

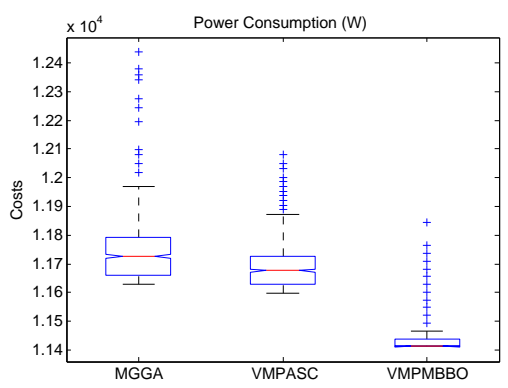

(c)

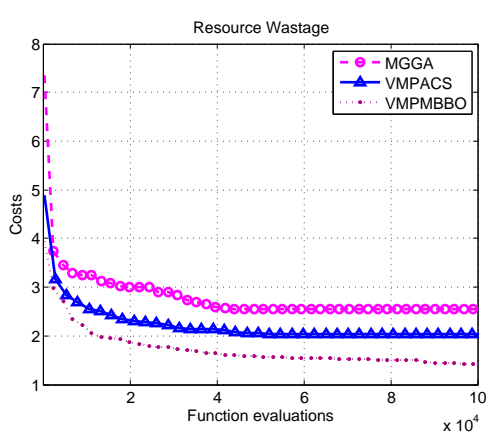

(b)

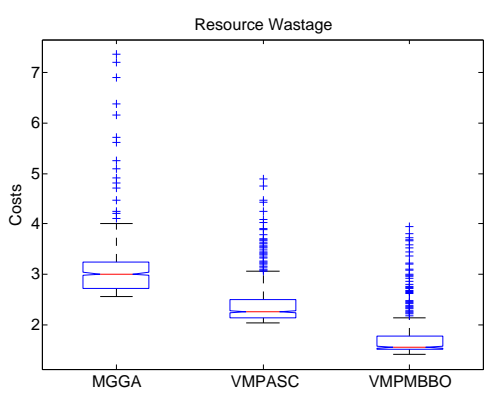

(d)

Fig. 9: Mean cost curve and boxplots of each objective in $\overline{D^{C P U}}=\overline{D^{M E M}}=25 \%$, Corr. $=-0.348$ 


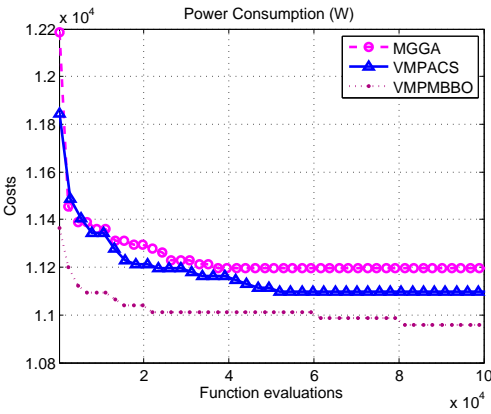

(a)

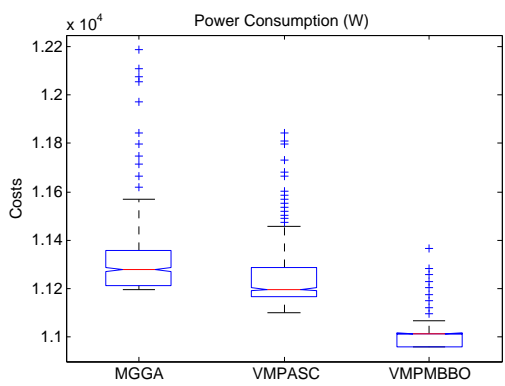

(c)

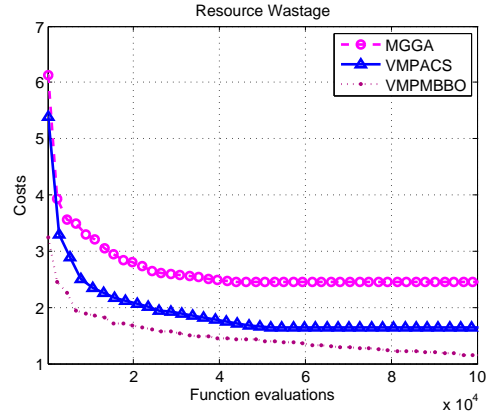

(b)

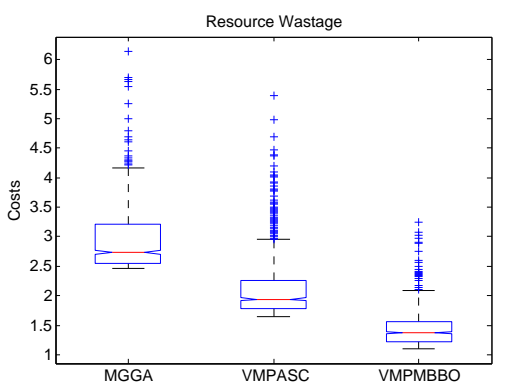

(d)

Fig. 10: Mean cost curves and boxplots of each objective in $\overline{D^{C P U}}=\overline{D^{M E M}}=25 \%$, Corr.$=-0.072$

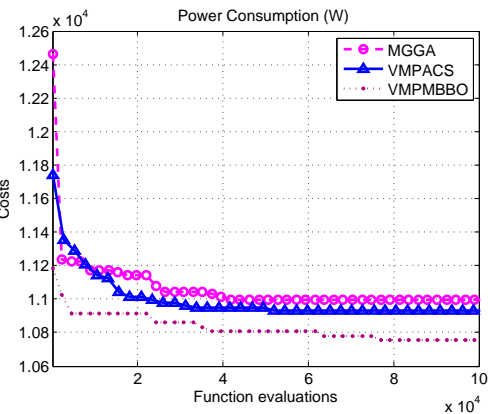

(a)

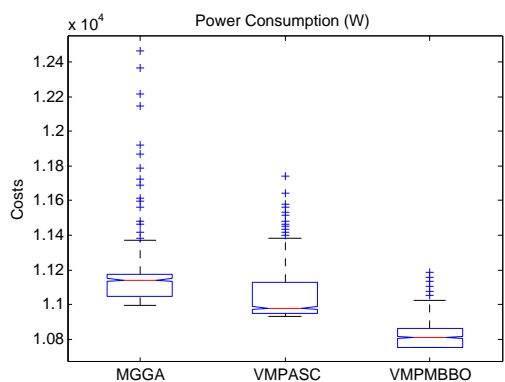

(c)

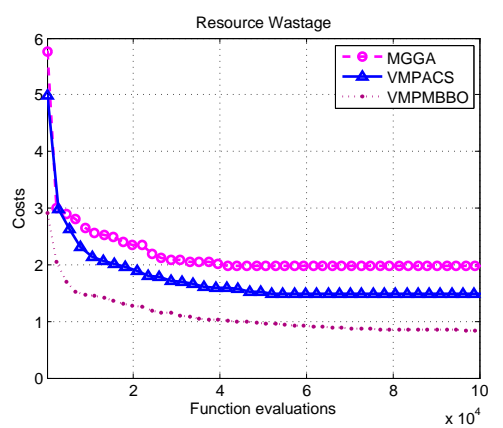

(b)

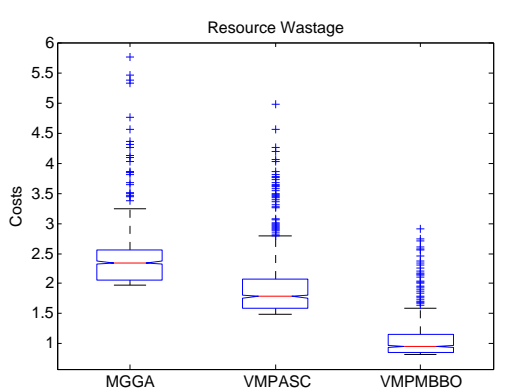

(d)

Fig. 11: Mean cost curves and boxplots of each objective in $\overline{D^{C P U}}=\overline{D^{M E M}}=25 \%$, Corr. $=0.371$ 


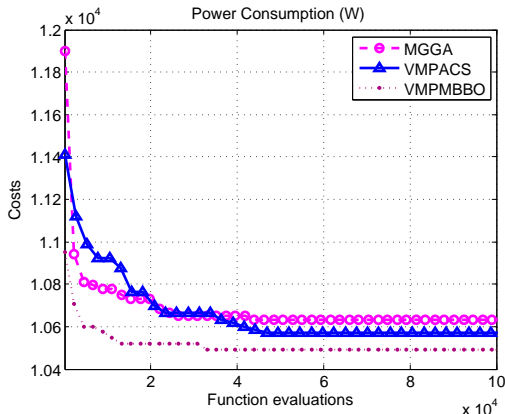

(a)

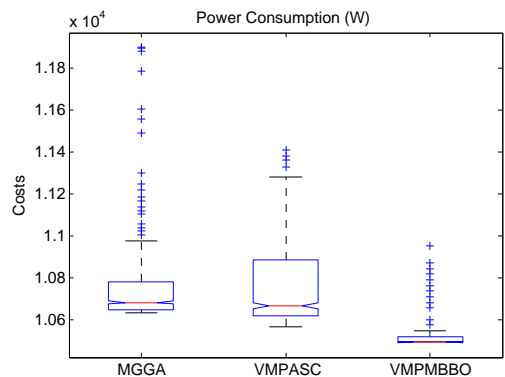

(c)

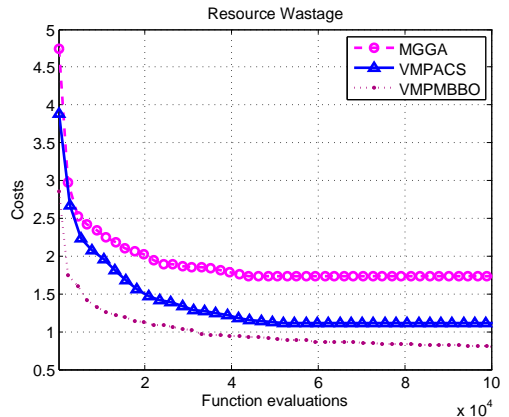

(b)

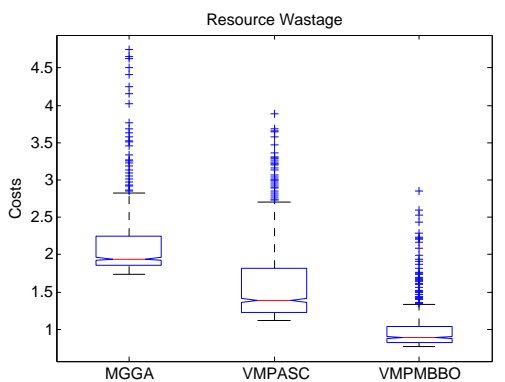

(d)

Fig. 12: Mean cost curves and boxplots of each objective in $\overline{D^{C P U}}=\overline{D^{M E M}}=25 \%$, Corr.$=0.755$

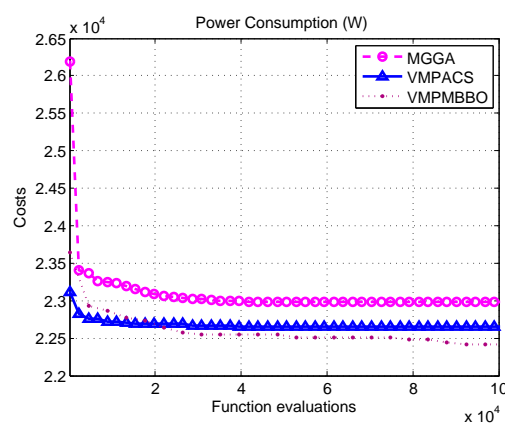

(a)

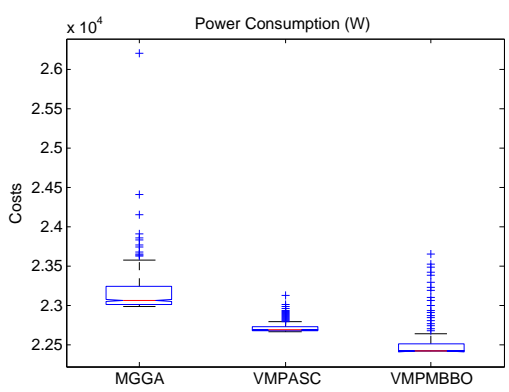

(c)

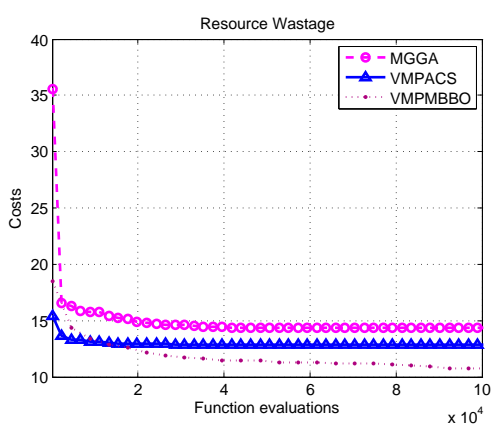

(b)

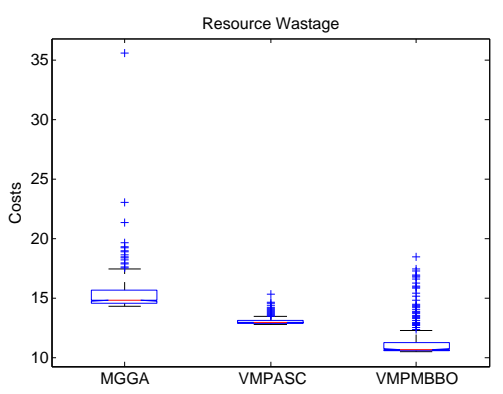

(d)

Fig. 13: Mean cost curve and boxplot of each objective in three algorithms with $\overline{D^{C P U}}=\overline{D^{M E M}}=45 \%$, Corr. $=-0.755$ 


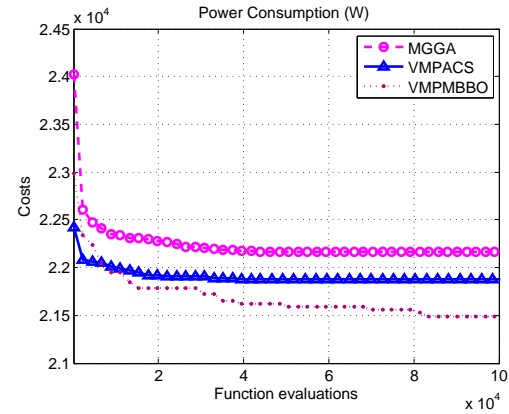

(a)

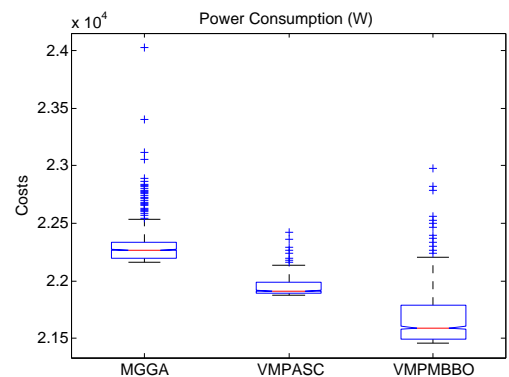

(c)

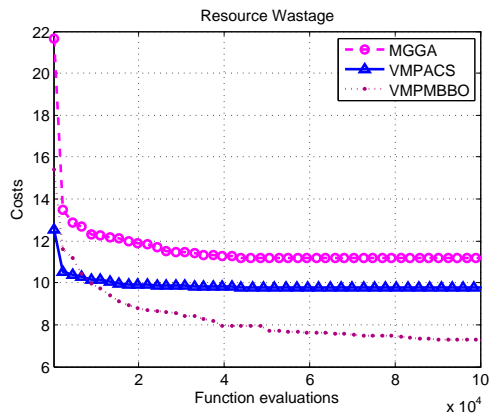

(b)

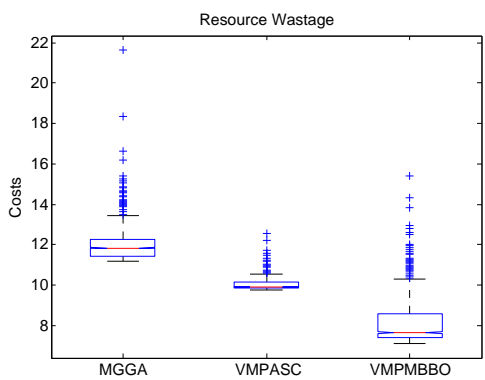

(d)

Fig. 14: Mean cost curves and boxplots of each objective in $\overline{D^{C P U}}=\overline{D^{M E M}}=45 \%$, Corr. $=-0.374$

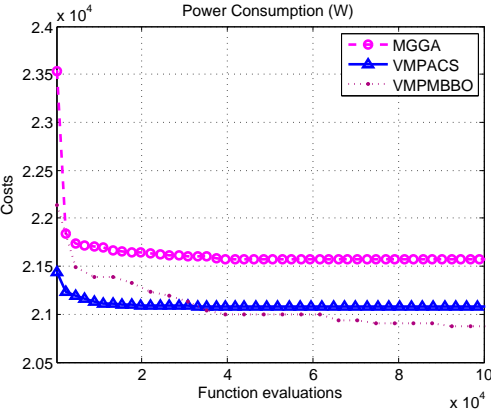

(a)

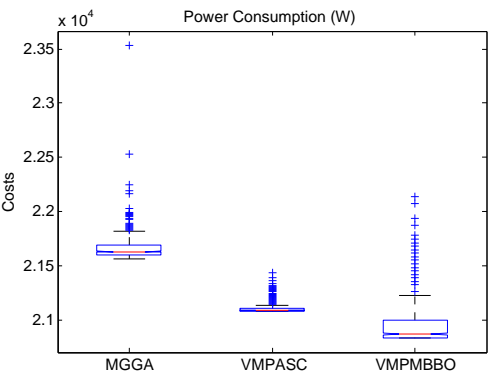

(c)

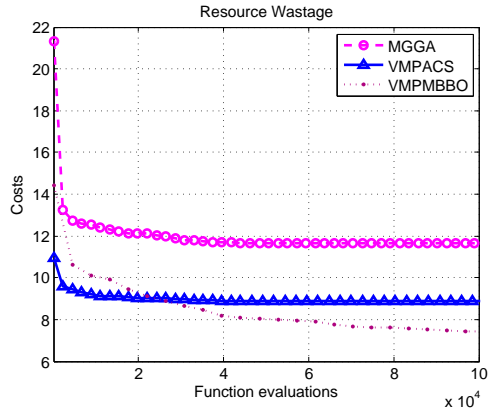

(b)

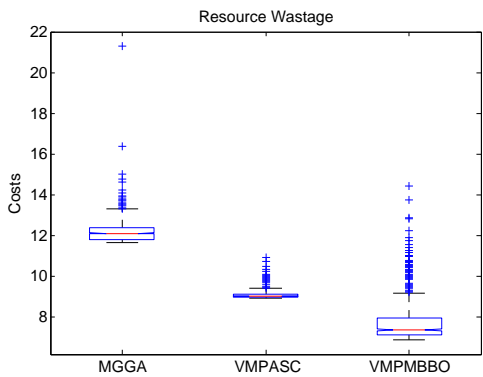

(d)

Fig. 15: Mean cost curves and boxplots of each objective in $\overline{D^{C P U}}=\overline{D^{M E M}}=45 \%$, Corr.$=-0.052$ 


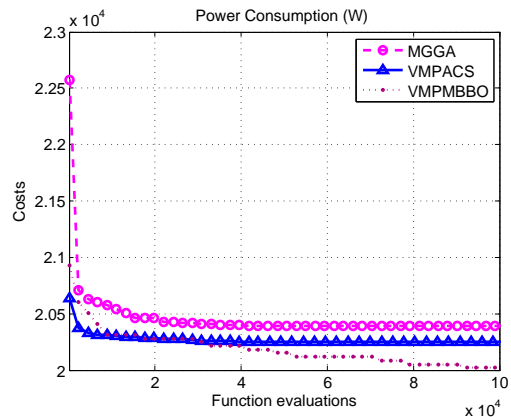

(a)

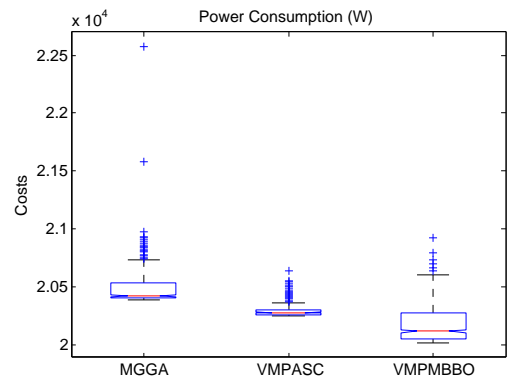

(c)

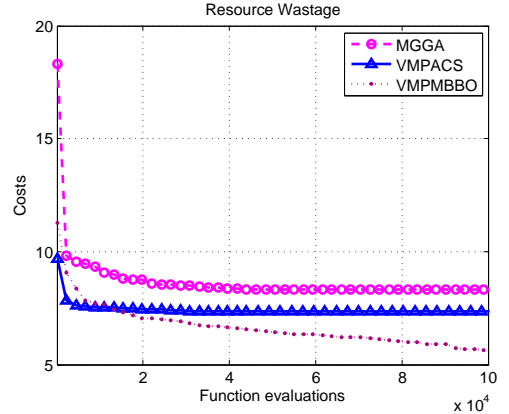

(b)

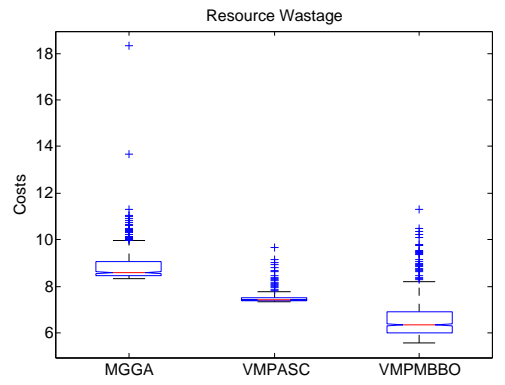

(d)

Fig. 16: Mean cost curves and boxplots of each objective in $\overline{D^{C P U}}=\overline{D^{M E M}}=45 \%$, Corr.$=0.398$

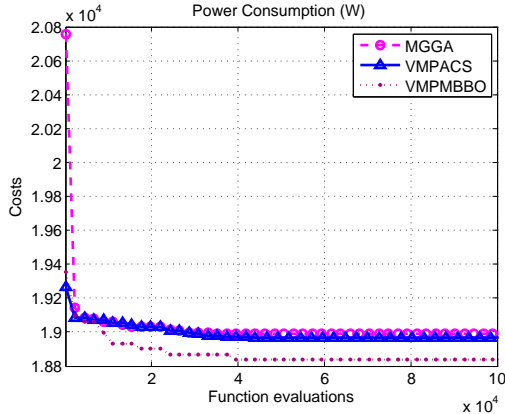

(a)

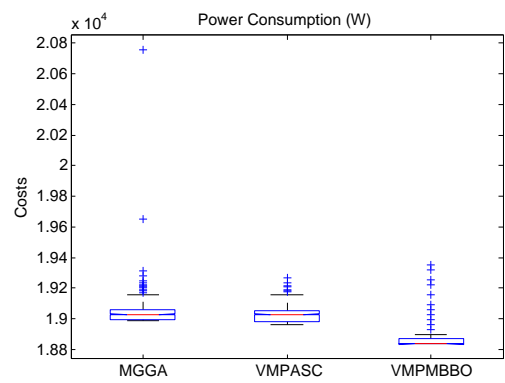

(c)

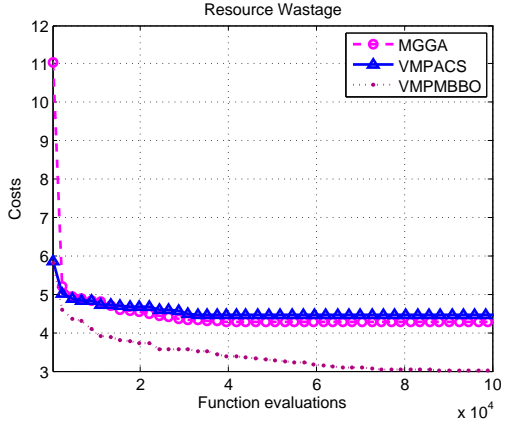

(b)

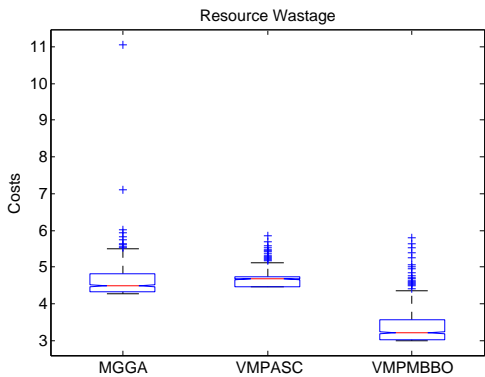

(d)

Fig. 17: Mean cost curves and boxplots of each objective in $\overline{D^{C P U}}=\overline{D^{M E M}}=45 \%$, Corr. $=0.751$ 


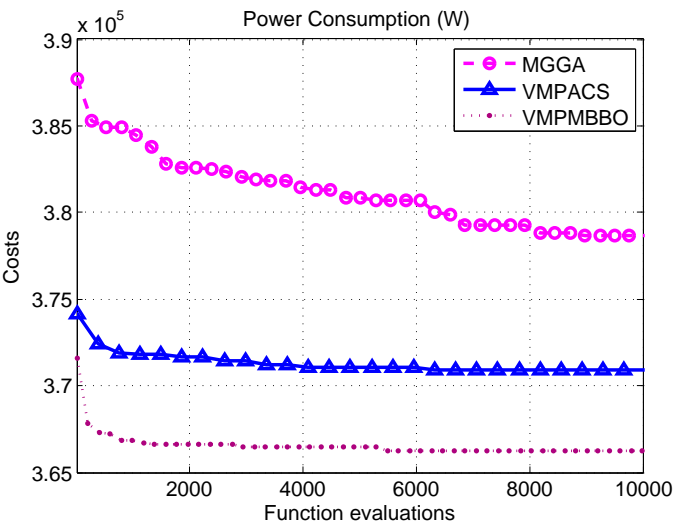

(a)

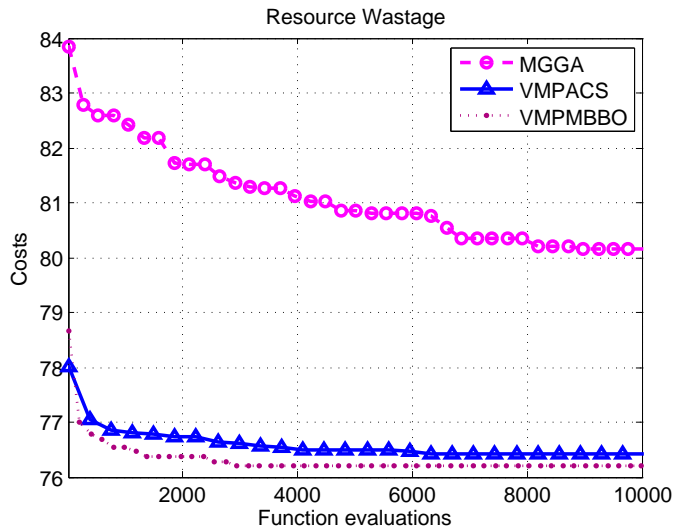

(b)

Fig. 18: Mean cost curve of each objective in three algorithms on Real Dataset-EC2 with 10,000 function evaluations

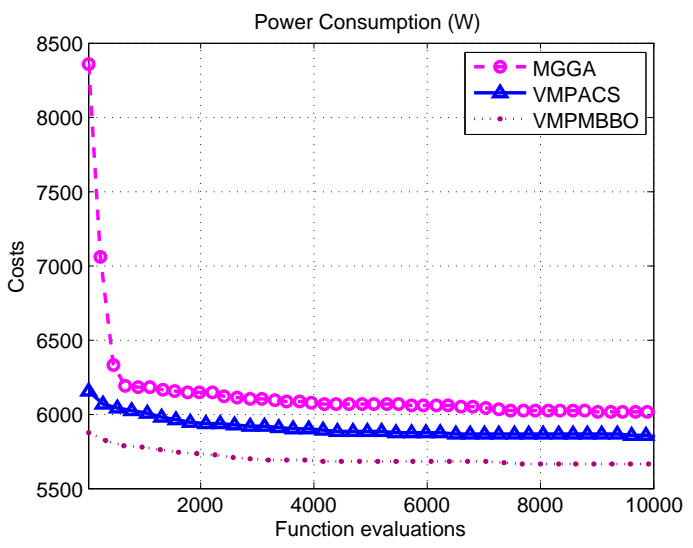

(a)

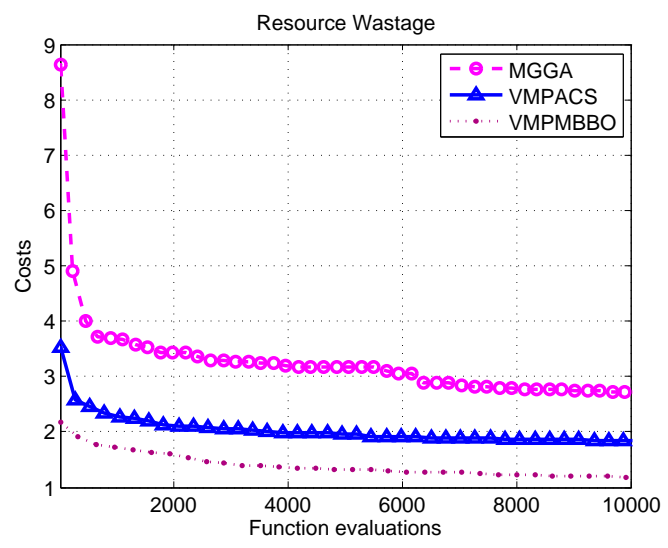

(b)

Fig. 19: Mean cost curve of each objective in three algorithms on Real Dataset-XJTUDLC with 10,000 function evaluations

\subsection{Computational Efficiency}

As VMcP problem is NP-hard, it is important to obtain approximate optimal solutions within an acceptable time period. In this section, the efficiency of the three VMcP algorithms, MGGA, VMPACS and VMPMBBO are compared and contrasted.

1) Dataset

Three types of datasets have been used in Scenarios 25-28. In Scenarios 25 and 26, twenty datasets have been generated using Algorithm 3. The dataset in Scenario 27 is the same as in Scenario 23, which is based on 8,000 VMs consolidated in VMcP II of Scenario 11. Real Dataset-XJTUDLC is used in Scenario 28, which is the same as was used in Scenario 24.

2) Result Evaluation

The run time refers to the time spent on searching for the approximate optimal solution, after 100,000 function evaluations on synthetic dataset and 10,000 function evaluations on real datasets. Scenario 25 shows how run time changes with varying number of VM requests from 200 to 2000 when $P=0.5, \overline{D^{C P U}}=\overline{D^{M E M}}=25 \%$, Corr. $=$ -0.072 . Each value, as plotted in Fig. 22(a) shows average run time of three repeated runs of each instance. The curves illustrate that the execution time of VMPMBBO is always less than MGGA. Compared to VMPACS, VMPMBBO has about same execution time when the number of VM requests is smaller than 800. After that, VMPMBBO always runs faster than VMPACS. Overall, VMPMBBO is computationally more efficient than MGGA and VMPACS as shown in 


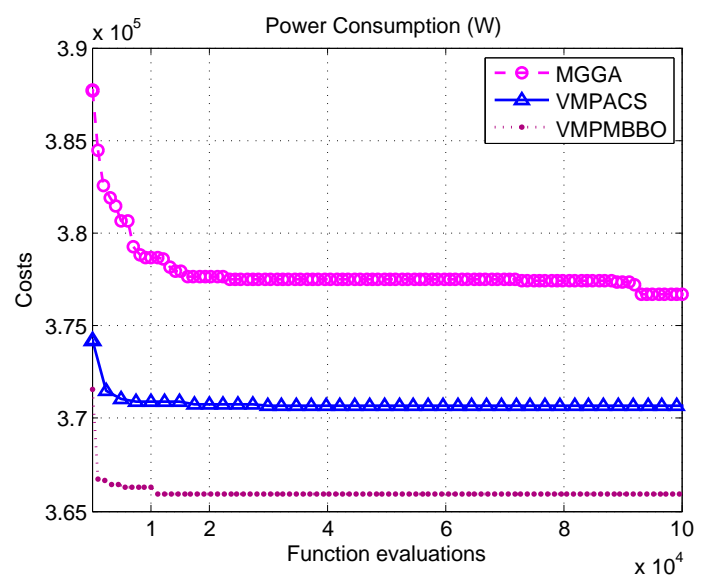

(a)

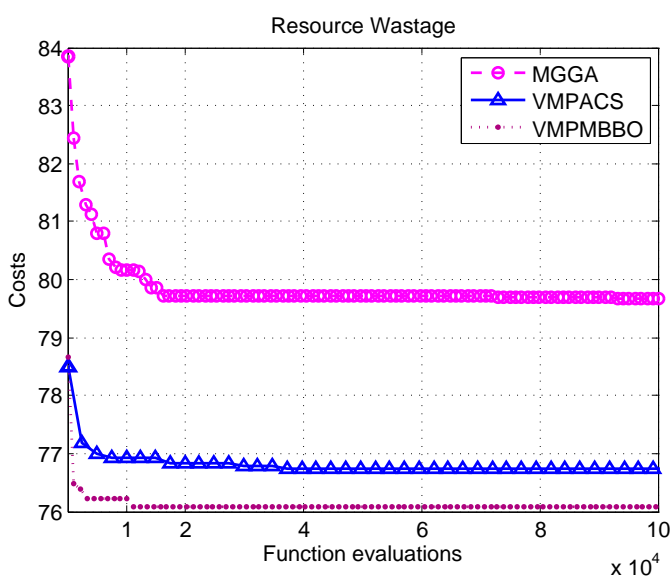

(b)

Fig. 20: Mean cost curve of each objective in three algorithms on Real Dataset-EC2 with 100,000 function evaluations

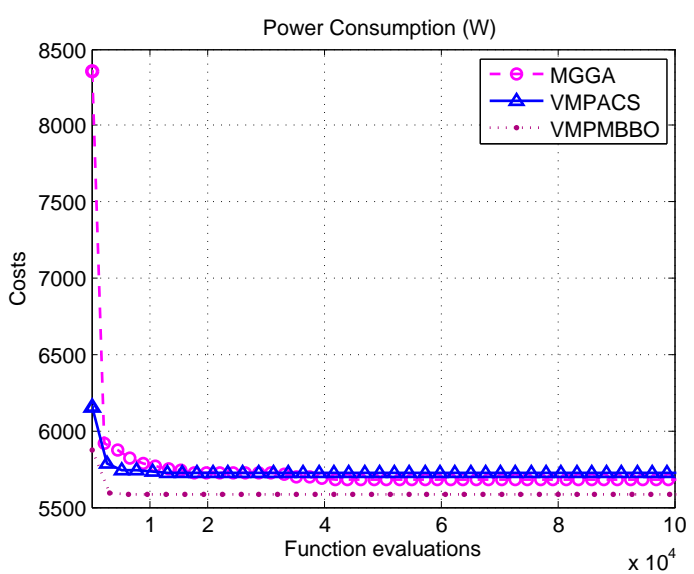

(a)

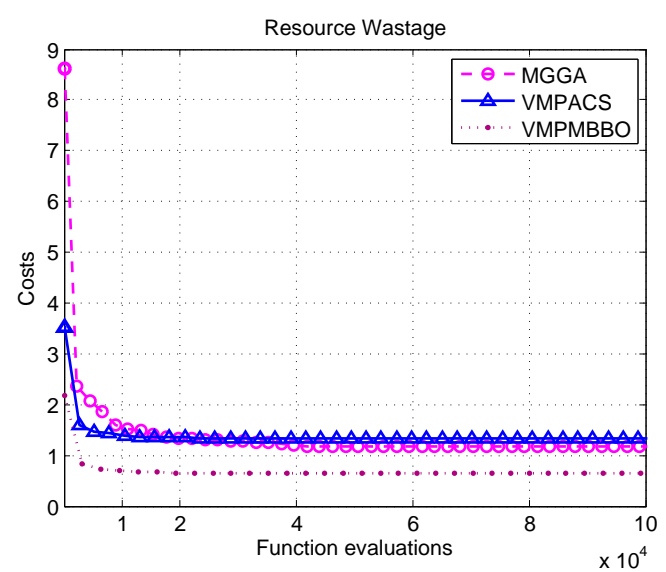

(b)

Fig. 21: Mean cost curve of each objective in three algorithms on Real Dataset-XJTUDLC with 100,000 function evaluations

\section{Scenario 25 .}

Fig. 22(b) for the scenario 26 also shows that VMPMBBO has a better computational efficiency when $P=0.5$, $\overline{D^{C P U}}=\overline{D^{M E M}}=45 \%$, Corr. $=-0.052$. Another observation is that when $\overline{D^{C P U}}=\overline{D^{M E M}}=25 \%$, it takes 213 seconds for VMPMBBO to obtain an approximate optimal solution for consolidating $1000 \mathrm{VMs}$, and 754 seconds for consolidating $2000 \mathrm{VMs}$, after 100,000 function evaluations. In contrast, when $\overline{D^{C P U}}=\overline{D^{M E M}}=45 \%$, it takes VMPMBBO 270 and 1112 seconds to obtain approximate optimal solutions of consolidating 1000 VMs and 2000 VMs respectively, after 100,000 function evaluations. The reason for this difference in results with the same number of new VMs is due to fact that the number of servers used to host VMs differs, on average there are four VMs per server in case of $\overline{D^{C P U}}=\overline{D^{M E M}}=5 \%$, and two VMs per server in the case of $\overline{D^{C P U}}=\overline{D^{M E M}}=45 \%$.

In Scenarios 25 and 26, as each VM is randomly generated with Algorithm 3. As almost no pattern can be found in these instances, the number of physical servers requested cannot be predicted correctly. The number of servers is set as the number of VMs in order to support the worst VM placement scenario (one VM per server) [30, 31]. For $\mathrm{VMcP}$ problem, the optimal solution may be unreachable in polynomial time, especially consolidating a large number of VMs to large number of VMs. It is obvious that reducing the number of optional servers will narrow search space and will reduce the run time.

The process of solving VMcP problems in large scale is a process of approximating optimal solution. Fig. 23 


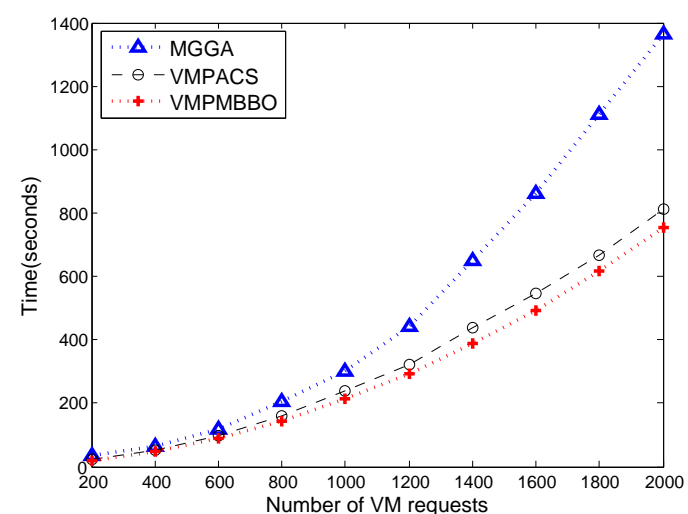

(a) $\overline{D^{C P U}}=\overline{D^{M E M}}=25 \%$, Corr. $=-0.072$

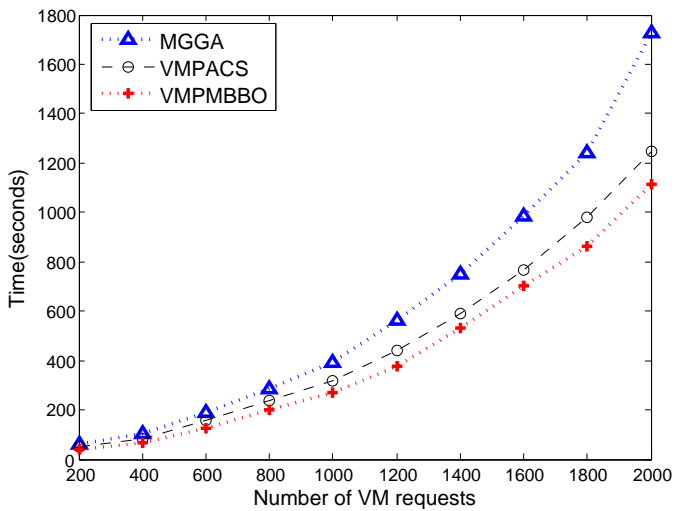

(b) $\overline{D^{C P U}}=\overline{D^{M E M}}=45 \%$, Corr. $=-0.052$

Fig. 22: Run times relative to the number of VM requests on synthetic dataset

shows how run time changes with function evaluations in the real scenarios (Scenarios 27 and 28).In Scenario 27, VMPMBBO takes 229 seconds to get the solution at $10,000^{\text {th }}$ function evaluations, when total power consumption has been reduced by more than 10 percent (details refer to Table 6). And VMPMBBO takes 13 seconds in Scenario 28. A total of 8,000 VMs have been consolidated in Scenario 27. However, the run time in Scenario 27 is significantly lower than in Scenarios 25 and 26, where 2,000 VMs have been relocated. The reason why VMPMBBO converges fast to an optimal solution in the real-world problem is that the number of instance types and the number of servers both are small and determined in the process of VMiP.

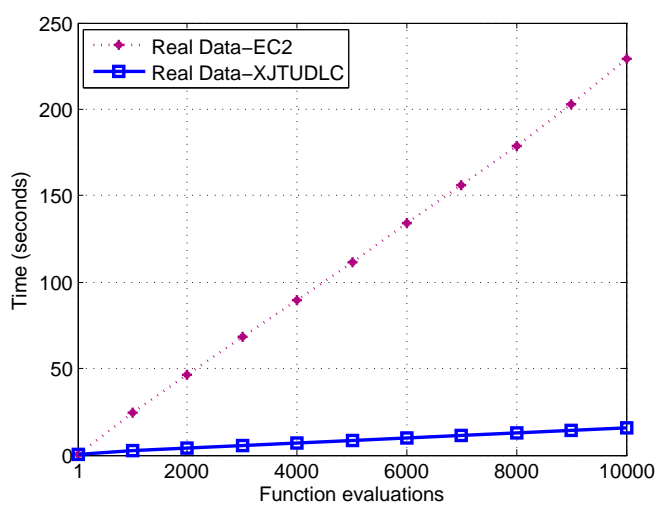

Fig. 23: Run times of VMPMBBO relative to the number of function evaluations on real datasets

\subsection{Robustness}

The robustness of the proposed VMPMBBO approach has been validated in Scenarios 29-31, in the sense that the performance obtained by setting different subsystems and popsize values does not vary considerably in most cases.

Synthetic dataset has been used in Scenarios 29-31, which is the same as in Scenarios 13-22.

Subsystems and popsize (i.e. number of islands per subsystem) are two key parameters of VMPMBBO. The intuition is that the performance will improve with more subsystems and larger subsystems, because there are more candidate solutions to explore and more generations of solutions get produced. To verify this hypothesis, we carried out some experiments. The experiments use sixteen pairs of Subsystems and popsize, as listed in Table 10.

In Scenario 29, we examine the performance under the different combinations of subsystems and popsize. Fig. 24 plots the power consumption and resource wastage after 100, 000 cost evaluations, under different combinations of subsystems and popsize. It can be seen that the curves of power consumption do not change much in these scenarios. 
This is because each SIV in an immigrating island has a chance to be replaced by a SIV from an emigrating island. And there is a linear relationship between power consumption and CPU utilization of a server. In contrast, due to lack of such a linear relationship between resource wastage and CPU utilization, the resource wastage fluctuates a lot in these scenarios.

Table 10: Different combinations of subsystems and popsize in our experimental tests

\begin{tabular}{ccccccccccccccccc}
\hline Test number & $\mathrm{f} 1$ & $\mathrm{f} 2$ & $\mathrm{f} 3$ & $\mathrm{f} 4$ & $\mathrm{f5}$ & $\mathrm{f6}$ & $\mathrm{f} 7$ & $\mathrm{f} 8$ & $\mathrm{f} 9$ & $\mathrm{f} 10$ & $\mathrm{f} 11$ & $\mathrm{f} 12$ & $\mathrm{f} 13$ & $\mathrm{f} 14$ & $\mathrm{f} 15$ & $\mathrm{f} 16$ \\
\hline Subsystems & 2 & 2 & 2 & 2 & 4 & 4 & 4 & 4 & 10 & 10 & 10 & 10 & 20 & 20 & 20 & 20 \\
Popsize & 3 & 5 & 10 & 20 & 3 & 5 & 10 & 20 & 3 & 5 & 10 & 20 & 3 & 5 & 10 & 20 \\
\hline
\end{tabular}

Next, we built Scenarios 30 and 31 to examine the performance when fixing the values of one of the two parameters: subsystems or popsize and see how VMPMBBO responds to changed values of the parameter. Figs. 25 and 26 depict how VMPMBBO responds to changing values of the other parameter. Fig. 25 depicts the mean cost curves for varying number of subsystems $(2,4,10,20)$ when popsize $=3, P=0.5$, and $\overline{D^{C P U}}=\overline{D^{M E M}}=25 \%$, Corr. $=-0.072$ or $\overline{D^{C P U}}=\overline{D^{M E M}}=45 \%$, Corr. $=-0.052$. Fig. 26 draws the mean cost curves for different popsizes $(3,5,10,20)$ when Subsystems $=4, P=0.5, \overline{D^{C P U}}=\overline{D^{M E M}}=25 \%$, Corr. $=-0.072$ or $\overline{D^{C P U}}=\overline{D^{M E M}}=45 \%$, Corr. $=-0.052$. For comparison purpose, the performances of MGGA and VMPACS are also included in Figs. 25 and 26. It is observed that the performances of VMPMBBO do not change much with more subsystems or larger popsizes. This is because there are few solution points available for VMPMBBO to evolve from. Another observation is that in most cases VMPMBBO can converge to optimal solutions more quickly than MGGA and VMPACS in all settings of these two parameters. These observations indicate that the robustness of VMPMBBO in sense that the performance is still competitive and does not vary considerably when adopting different parameter settings.
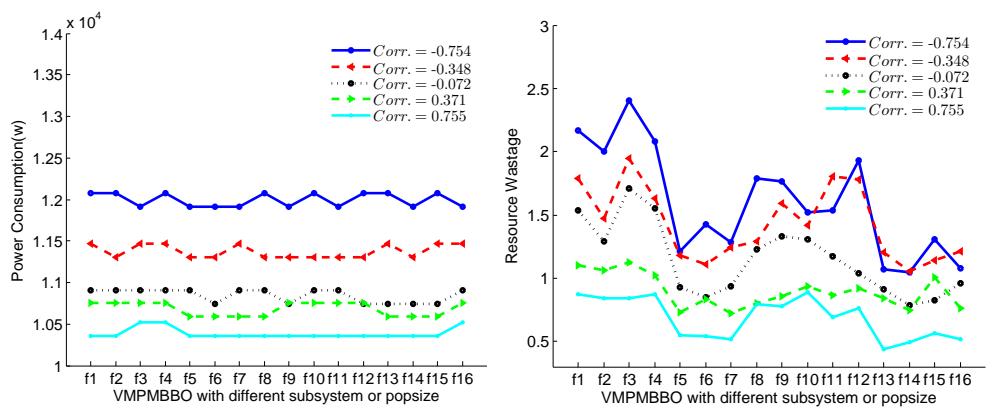

(a) in the case of $\overline{D^{C P U}}=\overline{D^{M E M}}=25 \%$
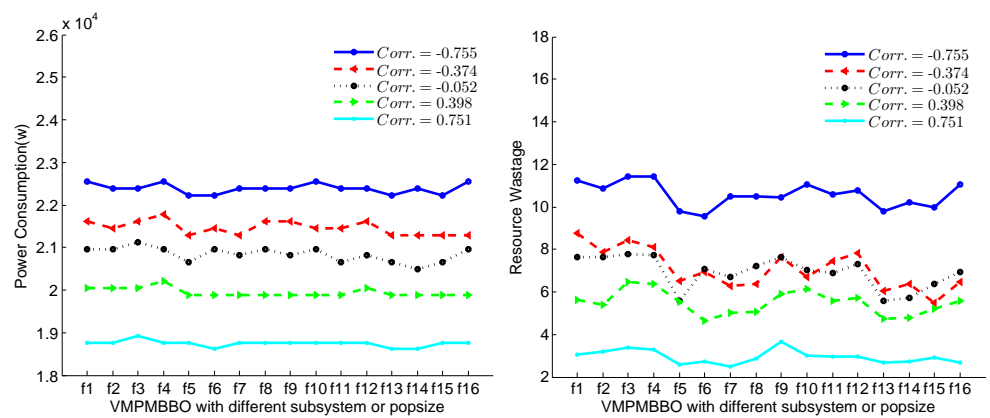

(b) in the case of $\overline{D^{C P U}}=\overline{D^{M E M}}=45 \%$

Fig. 24: Power consumption and resource wastage of VMPMBBO with different subsystems and popsize 

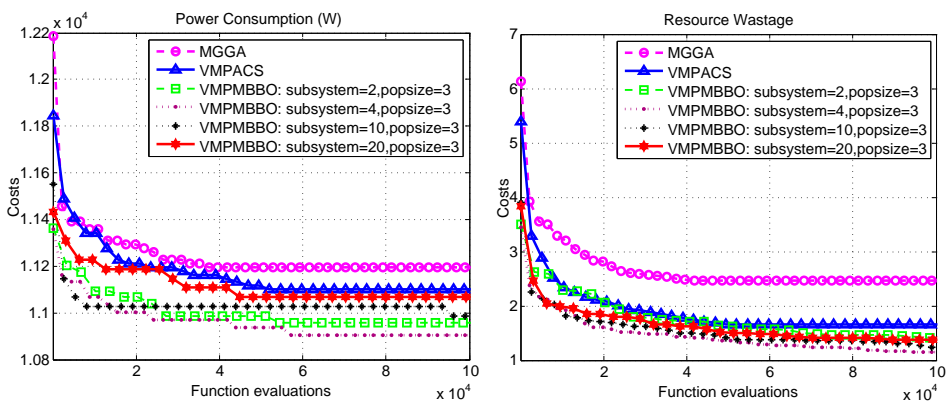

(a) in the case of $\overline{D^{C P U}}=\overline{D^{M E M}}=25 \%$, Corr.$=-0.072$
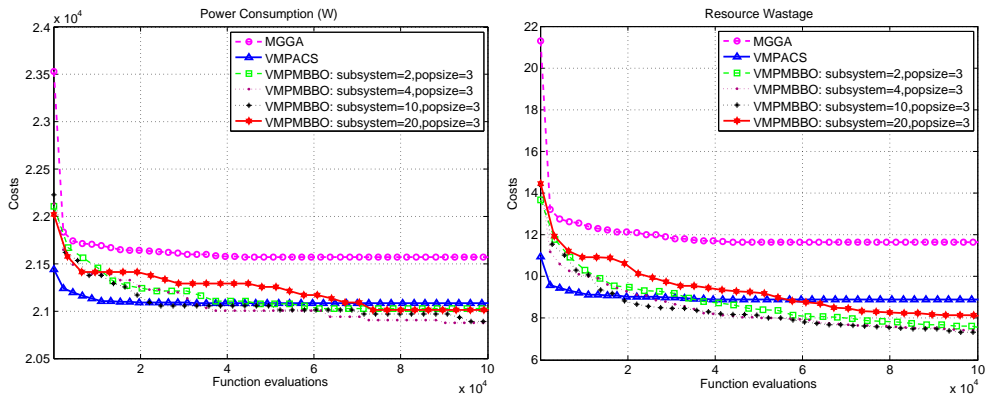

(b) in the case of $\overline{D^{C P U}}=\overline{D^{M E M}}=45 \%$, Corr.$=-0.052$

Fig. 25: Mean cost curves of objectives in VMPMMBBO with different subsystems
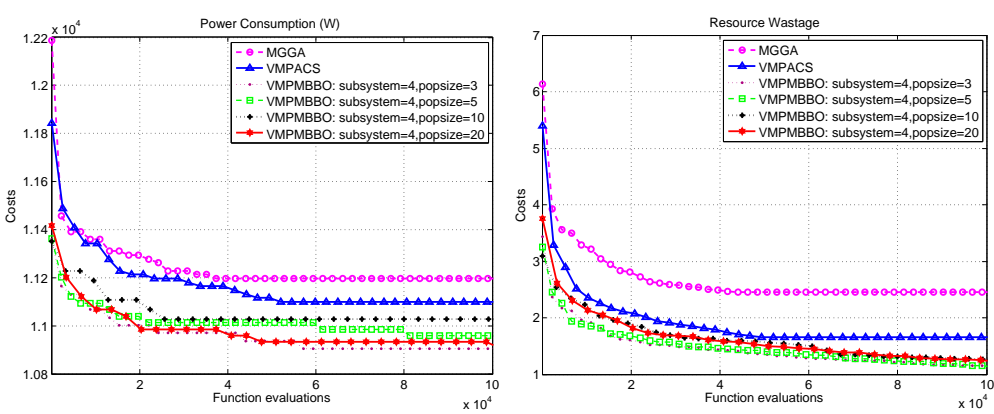

(a) in the case of $\overline{D^{C P U}}=\overline{D^{M E M}}=25 \%$, Corr . $=-0.072$
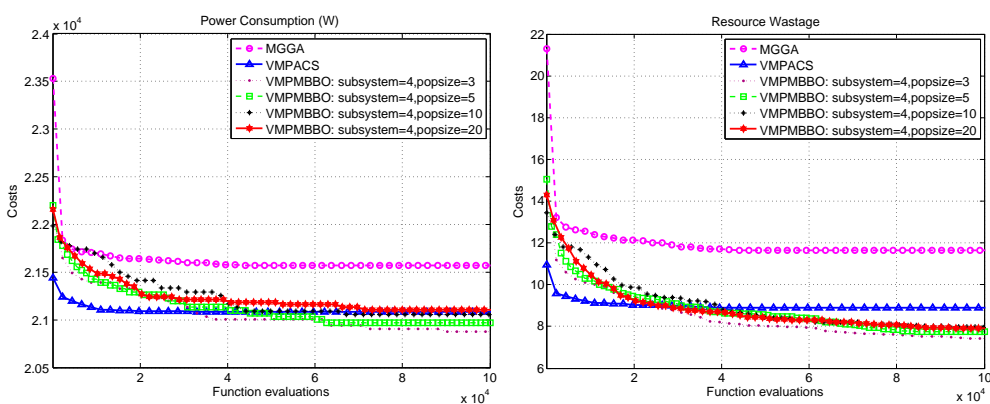

(b) in the case of $\overline{D^{C P U}}=\overline{D^{M E M}}=45 \%$, Corr.$=-0.052$

Fig. 26: Mean cost curves of objectives in VMPMMBBO with different popsizes 


\subsection{Parameter Value Selection}

In Scenarios 32-34, the selection of parameter values for VMPMBBO has been discussed, including mutation probability $P_{\text {mutation }}$, replacement probability $P_{\text {SIVmigration }}$, immigration rate $\lambda$ and emigration rate $\mu$.

Synthetic dataset has been used in Scenarios 32-34, which is the same as in Scenarios 13-22 .

- $P_{\text {mutation }}$ and $P_{\text {SIVmigration. }}$ Mutation rate $P_{\text {mutation }}$ and $S I V$ migration rate $P_{\text {SIVmigration }}$ are also important parameters of VMPMBBO. In BBO/Complex [39] the mutation rate of 0.05 is recommended. Experiments are conducted in order to find out the appropriate setting for these two parameters in VMPMBBO. Fig. 27 plots the statistical distributions of the power consumption and resource wastage with various mutation rates when $\overline{D^{C P U}}=\overline{D^{M E M}}=45 \%$, Corr. $=-0.052$. In each subfigure, the left most boxplot is for $P_{\text {mutation }}$ being 0 . The second boxplot from the left is for the mutation rate of 0.05 , the third from the left is for the mutation rate of 0.01. It is shown that the mutation does improve performance when the mutation rate $\left(P_{\text {mutation }}\right)$ is below 0.4 . The mutation rate $\left(P_{\text {mutation }}\right)$ of 0.05 has the best overall performance. Fig. 28 presents the changes of the two objective functions with different SIV migration rates. The result indicates that the power consumption is not dramatically affected by the SIV migration rate, but the resource wastage is least when the SIV migration rate is 0.5 . Based on these experiment results, we use the mutation rate of 0.01 and SIV migration rate of 0.5 for VMPMBBO.
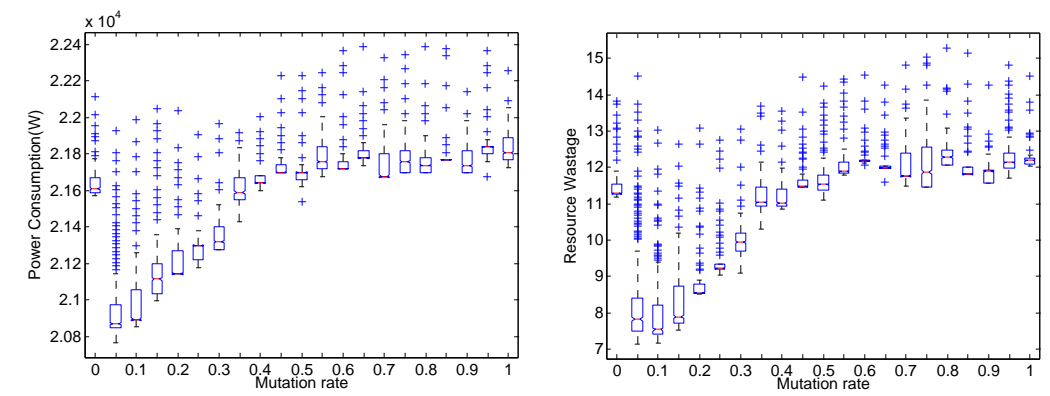

(a) $\overline{D^{C P U}}=\overline{D^{M E M}}=45 \%$, Corr. $=-0.052$

Fig. 27: Boxplots of each objective obtained by VMPMBBO with different mutation rates
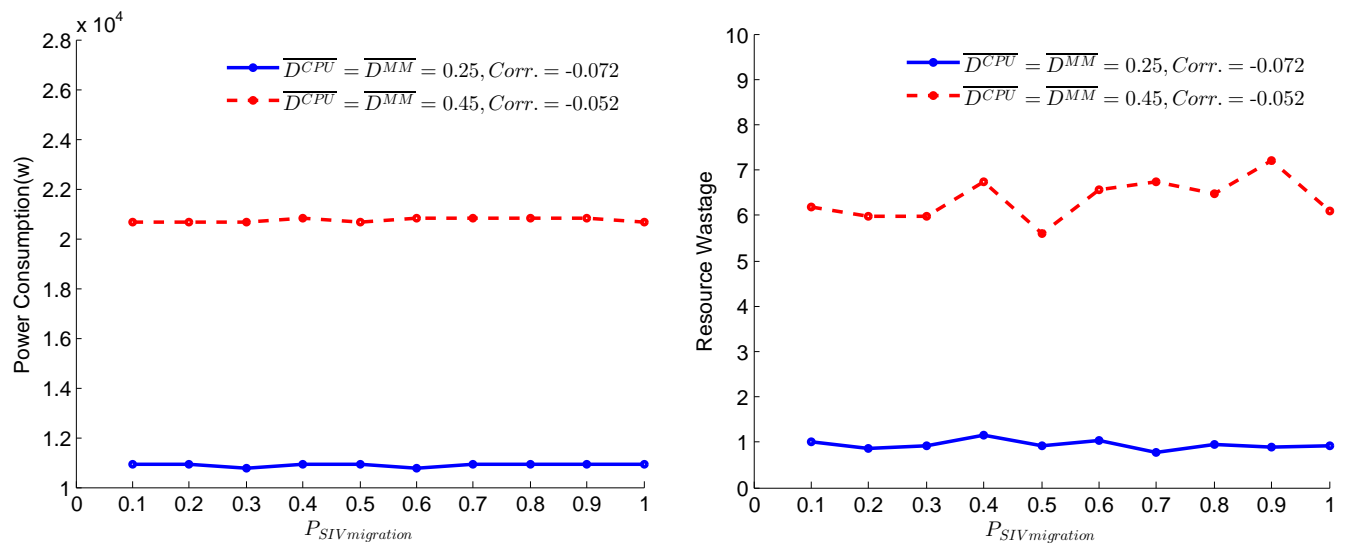

Fig. 28: The costs of VMPMBBO with different SIV migration rates

- $\lambda$ and $\mu$. The immigration and emigration curves are straight lines in the initial work of BBO [38]. It means that the immigration rate $\lambda$ and emigration rate $\mu$ are linear (Eq.12) functions of the number of species (i.e. the values of HSI). As mentioned earlier, other strategies such as quadratic in Eq.13, cosine in Eq.14, and 
exponential in Eq.15 have been proposed in [56, 57]. In order to find out which of these strategies and the strategy described in Eq.11 is the best for VMPMBBO. Experiments are carried out and the average cost curves of these strategies are presented in Fig. 29 for case of $\overline{D^{C P U}}=\overline{D^{M E M}}=25 \%$, Corr. $=-0.754$ and Fig. 30 for the case of $\overline{D^{C P U}}=\overline{D^{M E M}}=45 \%$, Corr . $=-0.052$. In these figures strategy 1 , strategy 2 , strategy 3 , strategy 4, and strategy 5 refer to quadratic in Eq.11, linear in Eq.12, quadratic in Eq.13, cosine in Eq.14, and exponential in Eq.15. The emigration rate $\mu$ is always $1-\lambda$. It reveals that the quadratic strategy in (11) converges to a lower minimum faster than the other four. The results in the other correlation scenarios support the same conclusion, but are not included in this paper due to the space limitation.

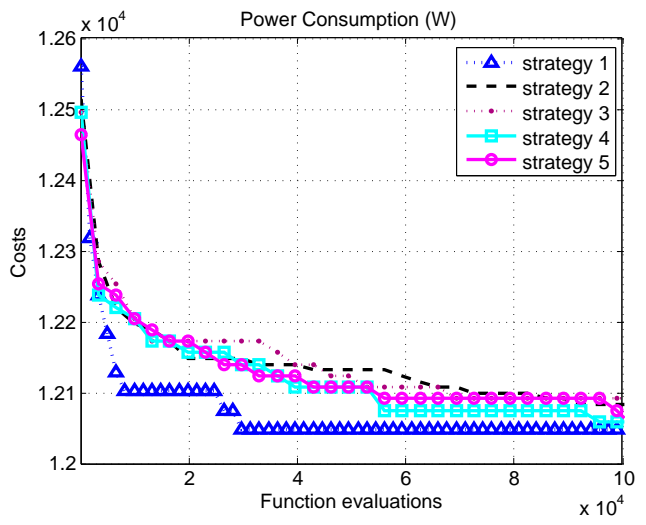

(a)

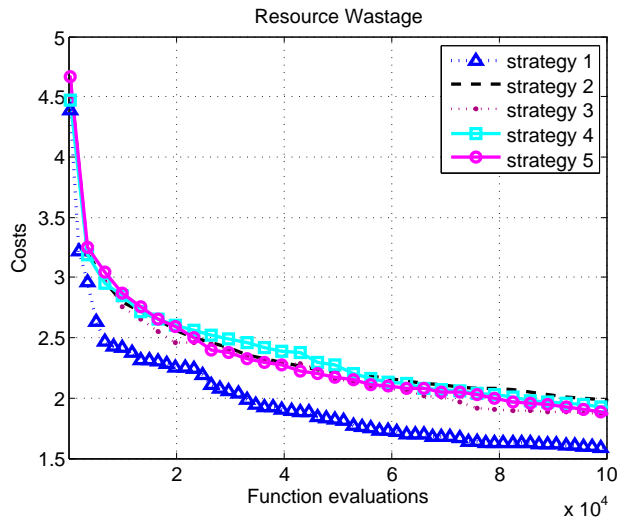

(b)

Fig. 29: Mean cost curves with five migration rate generation strategy in $\overline{D^{C P U}}=\overline{D^{M E M}}=45 \%$, Corr. $=-0.754$

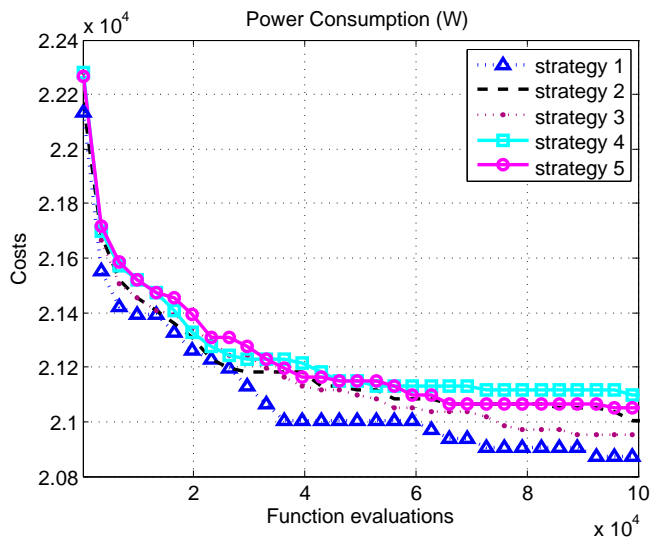

(a)

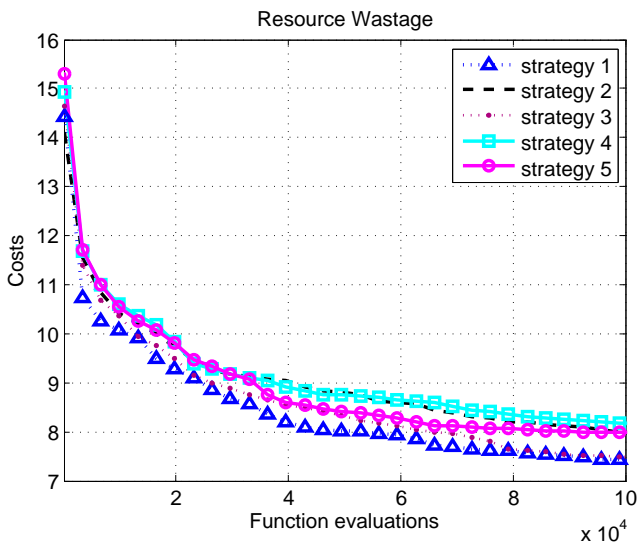

(b)

Fig. 30: Mean cost curves with five migration rate generation strategy in $\overline{D^{C P U}}=\overline{D^{M E M}}=45 \%$, Corr. $=-0.052$

\section{Adaptability and Extensibility of VMPMBBO}

In VMPMBBO, VMcP problem has been solved as a multi-objective optimization problem. As mentioned in Section 4, the feasible solutions of each objective have been obtained from different subsystems. The subsystems are loosely coupled and they communicate with each other by cross-subsystem migration. Therefore, VMPMBBO can easily solve more complex problems involving more objectives or constraints. 
In the following subsections, the adaptability and extensibility have been proved by using a complex scenario, where the optimization model incorporates many factors, including resource wastage, power consumption, server load balance [44, 61, 62, 45, 46, 47], Inter-VM network traffic [41, 42], storage traffic [63] and anti-affinity policy $[64,65,66]$.

\subsection{Extended Model}

Five objectives have been set in this VMcP scenario to reduce power consumption (Eq.3), reduce resource wastage (Eq.4), balance the server loads (Eq.16), reduce inter-VM network traffic due to the inherent dependencies (Eq.17), and also reduce storage traffic by storage affinity policy(Eq.18). Moreover, both VM-to-PM mapping and VM-to-SN mapping have been considered in the extended VMcP Model. Two kinds of VM-to-VM policies: affinity and antiaffinity policies $[64,65,66]$ have been considered. A kind of Server-to-Storage policy: storage affinity policy [63] has also been considered.

1) Server load balance model. Imbalance of servers is represented by the server load unevenness as follows, which has been adopted from [44, 61, 62, 45, 46, 47]:

$$
\text { unevenness }=\sum_{\phi=1}^{R} \sum_{j=1}^{M}\left|D_{j}^{\phi}-\mu\right|
$$

where $M$ is the number of used servers. $D_{j}^{\phi}$ is the load of resource $\phi$ on $j$-th server, and $\mu$ is the average load of resource $\phi$ on $M$ servers. $R$ is the number of resource dimension we have considered.

2) Inter-VM network traffic model. It is represented by the product of communication distance and inter-VM network bandwidth usage $[41,42]$ and modelled as follows:

$$
\text { NetworkTraffic inter-VM }=\sum_{i_{1}=1}^{N} \sum_{i_{2}=1}^{N}\left(\operatorname{Distance}\left(x_{i_{1}}, x_{i_{2}}\right) \cdot \operatorname{Usage}\left(i_{1}, i_{2}\right)\right) \text {, }
$$

where $N$ is the number of VMs. The value of variable $x_{i_{1}}$ indicates the server number assigned to $i_{1}$-th VM . $\operatorname{Distance}\left(x_{i_{1}}, x_{i_{2}}\right)$ is defined as the latency, delay or number of hops between server $x_{i_{1}}$ and $x_{i_{2}}$. Distance $\left(x_{i_{1}}, x_{i_{2}}\right)=0$ when $x_{i_{1}}=x_{i_{2}}$.Usage $\left(i_{1}, i_{2}\right)$ is defined as traffic demand between $i_{1}$-th VM and $i_{2}$-th VM.

3) Storage traffic model. It is assumed that the main storage is a distributed object storage system like Ceph [67]. Each storage node $(\mathrm{SN})$ has been coupled with a certain number of servers as a group, considering the storage affinity policy [63]. Each VM instance runs on a server and its image is stored in a storage node. If the servers where VMs are running and the storage node where VM images are stored are in the same group, the traffic between the servers and the storage system would be low. The storage traffic is modelled as follows:

$$
\text { StorageTraffic }=\sum_{i=1}^{N}\left(\operatorname{Distance}\left(x_{i}, x_{i}^{s n}\right) \cdot \text { StorageBandwidthUsage }(i)\right) \text {, }
$$

where the value of variable $x_{i}^{s n}$ indicates the storage node number assigned to $i$-th VM. Distance $\left(x_{i}, x_{i}^{s n}\right)$ is defined as latency, delay or number of hops between server $x_{i}$ and storage node $x_{i}^{s n}$. Distance $\left(x_{i_{1}}, x_{i_{2}}\right)=0$ when server $x_{i}$ and storage node $x_{i}^{s n}$ are in the same group. StorageBandwidthU sage $(i)$ is defined as storage traffic of VM $i$.

4) Constraint by anti-affinity policy. Affinity and anti-affinity policies have been widely used in virtualization infrastructure $[64,65,66]$. Affinity policies keep VMs together on the same server for reducing traffic across networks, which is represented as the objective in Eq.17. Anti-affinity policies separate VMs on different hosts for failover, which is considered as a constraint of $\mathrm{VMcP}$ model and specified as follows:

$$
\left|x_{i_{1}}-x_{i_{2}}\right|>0, \quad \forall i_{1}, i_{2} \in V M S e t^{\text {anti-Affinity }} \text { and } i_{1} \neq i_{2},
$$

where VMS et $t_{\text {AntiAffinity }}$ is a set of VMs restricted by anti-affinity policy.

5) Extended VMcP Model. The VMcP optimization problem has been extended as follows: Suppose that there are $N$ VMs which are to be placed on $M$ physical machines, the VM images are to be stored in $S$ storage node, and none of the VMs requires more resources than a single server or a storage node can offer. The resource allocation 
requests for a VM and the resource capacity of a PM (server) are both represented by a multi-dimensional vector. Each dimension refers to the amount of a specific type of resources requested by a VM or owned by a server or owned by a storage node. The aim is to simultaneously minimize power consumption, resource wastage, server load unevenness, inter-VM network traffic and storage traffic.

Goal:

$$
\begin{aligned}
& \text { Minimize: } \sum_{j=1}^{M} \text { resourceWastage }(j) \\
& \text { Minimize: } \sum_{j=1}^{M} \text { powerConsumption }(j) \\
& \text { Minimize: unevenness } \\
& \text { Minimize: NetworkTraffic } \text { inter-VM }^{\text {Minimize: StorageTraffic }}
\end{aligned}
$$

Constraints:

$$
\begin{aligned}
& x_{i} \in\{i, \ldots, M\} \quad i=[i, \ldots, N] \\
& x_{i}^{s n} \in\{1, \ldots, S\} \quad i=[1, \ldots, N] \\
& \sum_{i=1}^{N} D_{i}^{\text {storageS pace }} \cdot\left(x_{i}^{s n} \mid s\right) \cdot\left(s \mid x_{i}^{s n}\right) \leq T_{s}^{\text {storageS pace }} \cdot S N_{s} \quad s=[1, \ldots, S] \\
& \sum_{i=1}^{N} D_{i}^{r} \cdot\left(x_{i} \mid j\right) \cdot\left(j \mid x_{i}\right) \leq T_{j}^{r} \cdot y_{j} \quad j=[1, \ldots, M] \\
& \quad r=[C P U, \text { memory, extranet bandwidth, intranet bandwidth }] \\
& \sum_{i=1}^{N}\left(D_{i}^{\text {storageBandwidth }}\right) \cdot\left(x_{i}^{\text {sn }} \mid s\right) \cdot\left(s \mid x_{i}^{\text {sn }}\right) \leq T_{s}^{\text {storageBandwidth }} \cdot S N_{s} \quad s=[1, \ldots, S] \\
& \sum_{i=1}^{N}\left(\begin{array}{l}
\left.D_{i}^{\text {storageBandwidth }}\right) \cdot\left(x_{i} \mid j\right) \cdot\left(j \mid x_{i}\right) \leq T_{j}^{\text {storageBandwidth }} \cdot y_{j} \quad j=[1, \ldots, M] \\
S N_{s}=\left\{\begin{array}{l}
1, \quad \exists x_{i}^{s n}=s \\
0, \quad \text { others }
\end{array} \quad s=[1, \ldots, S]\right. \\
y_{i}=\left\{\begin{array}{l}
1, \quad \exists x_{i}=j \\
0, \quad \text { others }
\end{array} \quad j=[1, \ldots, M]\right. \\
\left|x_{i_{1}}-x_{i_{2}}\right|>0, \quad \forall i_{1}, i_{2} \in V M S e t^{\text {anti-Affinity }} \text { and } i_{1} \neq i_{2}
\end{array}\right.
\end{aligned}
$$

where $S$ is the number of storage nodes. The binary variable $S N_{s}$ indicates whether storage $s$ is used (value 1) or not (value 0$). D_{i}^{\text {storageS pace }}$ and $D_{i}^{\text {storageBandwidth }}$ refer to the space and storage bandwidth demand of $i$-th VM . Let $T_{s}^{\text {storageS pace }}$ be the threshold of the utilization of storage space. Let $T_{j}^{\text {storageBandwidth }}$ and $T_{s}^{\text {storageBandwidth }}$ be the threshold of storage bandwidth at a server side and at a storage node side respectively. Moreover, VM demands on CPU, memory, extranet bandwidth, intranet bandwidth and storage network bandwidth have been considered in resource wastage model presented in Eq. 20.

Constraints (Eqs.27-30) guarantee allocated resources from each server or storage node not to exceed their capacity. Constraint (Eq.31) defines the range of the variable . Constraint (Eq.33) ensures anti-affinity policy.

\subsection{Experiment Configuration}

Test Dataset. We consider the dataset where items independently follow normal distribution, which has been adopted in previous researches $[68,69,70]$. We generated six VM demand sets with normal distribution. We simulate 
a system with 50 servers, 10 storage nodes and 200 VMs. We use random VM-to-PM mapping and random VM-to-SN mapping in initial layout. CPU, memory and extranet bandwidth demands generated with $N(0.15,0.05)$. The storage bandwidth and storage space demands are generated with $N(0.03,0.01)$. Then, we randomly select $50 \%$ of VMs and pair them off. Let the VMs in $50 \%$ of the pairs be restricted by anti-affinity policy. And let inter-VM data movement take place in each of the other pairs. The inter-VM network traffic in each pair is generated with $N(0.15,0.05)$.

Network Configuration. Three networks have been considered in this scenario, including intranet, extranet and storage network. And they are fairly isolated from each other .The communication distance between servers is dependent on the architecture and is computed as shown in [42]. We use Tree architecture [71] to compute the distances both between server and storage node in storage network.

We have set up five subsystems, and make each subsystem correspond to an optimizing objective. The termination criterion is 10,000 function evaluations. Other configurations are described in Section 5.

\subsection{Result}

Fig. 31 plots the graph of function evaluations vs. each cost with error bars specified by the upper and lower edges of the shadow. In Fig. 31(c), the lower and upper edges of the shadow contain lower and upper error ranges for server unevenness. The white curves in Fig. 31 show the mean of power consumption, resource wastage, server unevenness and inter-VM network traffic at each function evaluation. The fluctuations in the white curves present the optimal decisions in the presence of trade-offs between conflicting objectives.

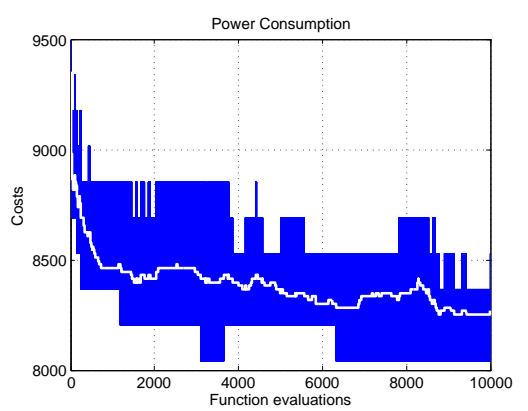

(a)

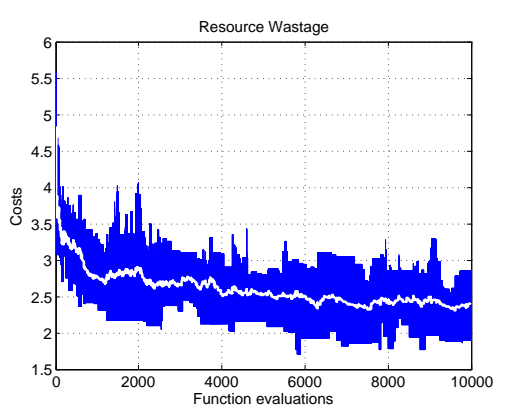

(b)

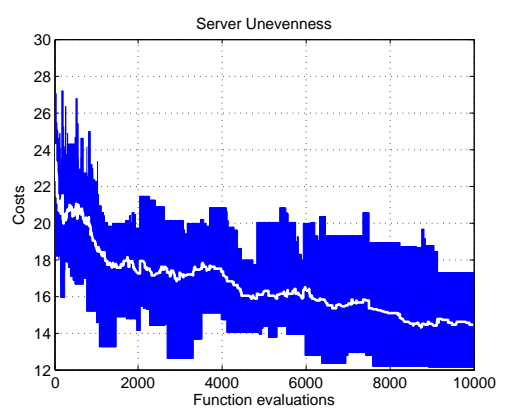

(c)

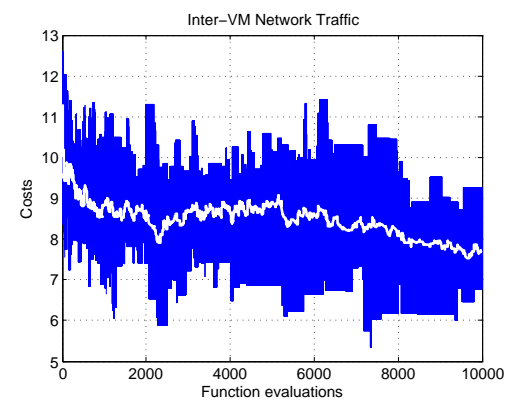

(d)

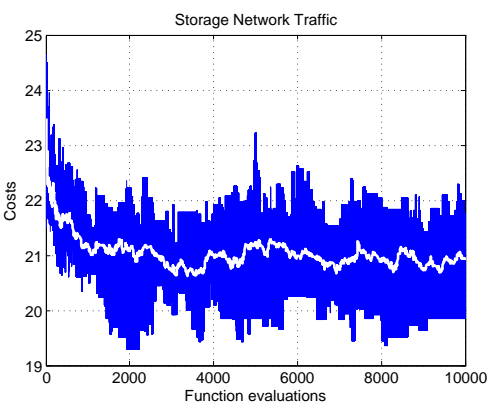

(e)

Fig. 31: Errorbars of VMPMBBO applied in extended VMcP model

Fig. 32 shows the CPU, memory and network bandwidth utilization of 50 servers before applying VMPMBBO, which is randomly generated as described in Section 6.4. Fig. 33 shows the resource utilization after applying VMPMBBO. As shown in Fig. 33, a total of 8 servers have been turned off for energy conservation. The load on servers are balanced, and for each server, the multi-dimensional resources are in a good equilibrium

\section{Conclusion and Future Work}

In this paper, we propose a novel VMcP solution called VMPMBBO. VMPMBBO treats VMcP problem as a complex system, and uses a biogeography based optimization method to optimally solve VMcP problem. The 


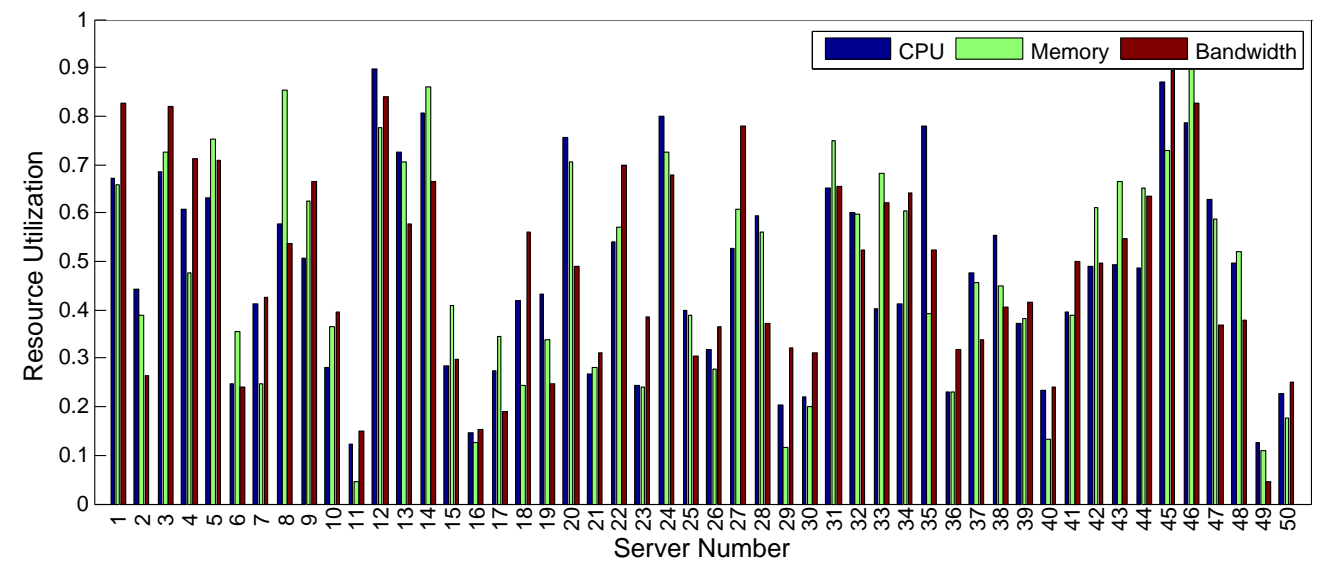

Fig. 32: Resource utilization of 50 servers before VMPMBBO

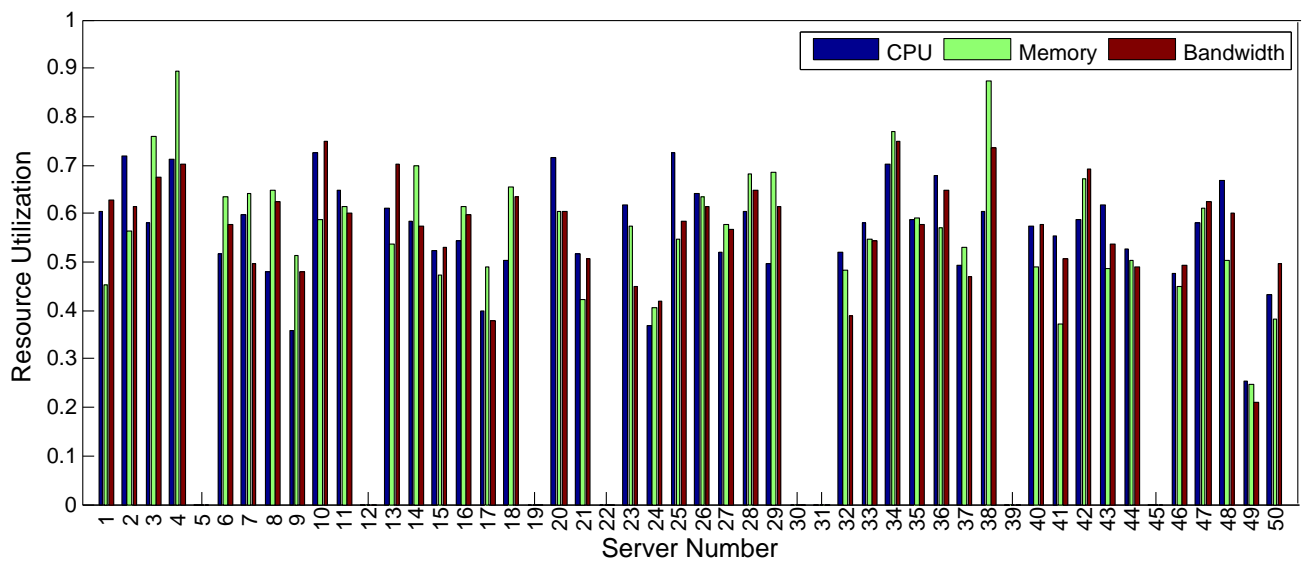

Fig. 33: Resource utilization of 50 servers after VMPMBBO

proposed VMcP solution optimizes multiple objectives such as power consumption and resource wastage at the same time. VMPMBBO is tested using both synthetic data from related literature and real data from two real-world data centers.

We conducted extensive simulations, evaluated the different parameter settings of the proposed approach and comparing it with two existing multi-objective VMcP solutions, MGGA and VMPACS. The experimental results show that in most cases, VMPMBBO has better convergence characteristics and it is computationally more efficient than MGGA and VMPACS. Adaptability and extensibility of VMPMBBO have also been proved. Future work will focus on parallelization of VMPMBBO.

\section{Acknowledgments}

This research was partially supported by National Science Foundation of China under Grant Nos. 91118005, 91218301, 61221063, 61103160 and 61103239, 61472315, the National Key Technologies R\&D Program of China under Grant No. 2012BAH16F02, Cheung Kong Scholar's Program, the Ministry of Education Innovation Research Team No. IRT13035, the MOE-Intel Special Research Foundation of Information Technology under Grant No. MOE-INTEL-2012-04, the Shanghai Special Foundation of Software and Integrated Circuit Industry under Grant No. 120421, and China Scholarship Council. Creative Project in Shanghai Science and Technology Council under Grant No.12dz1506200. 


\section{References}

[1] P. Mell, T. Grance, The nist definition of cloud computing, National Institute of Standards and Technology 53 (6) (2009) 50.

[2] W. Voorsluys, J. Broberg, R. Buyya, Introduction to cloud computing, Cloud Computing (2011) 1-41.

[3] T. Liu, T. Lu, W. Wang, Q. Wang, Z. Liu, N. Gu, X. Ding, Sdms-o: A service deployment management system for optimization in clouds while guaranteeing users qos requirements, Future Generation Computer Systems 28 (7) (2012) 1100-1109.

[4] S. G. M. R. A. K. P. S. [3] D. Filani, J. He, R. Nagappan, Technology with the environment in mind-dynamic data center power management: Trends, issues, and solutions, Intel Technology J. 1 (12) (2008) 59-68.

[5] J. Koomey, Growth in data center electricity use 2005 to 2010, A report by Analytical Press, completed at the request of The New York Times.

[6] P. Barham, B. Dragovic, K. Fraser, S. Hand, T. Harris, A. Ho, R. Neugebauer, I. Pratt, A. Warfield, Xen and the art of virtualization, SIGOPS Oper. Syst. Rev. 37 (5) (2003) 164-177. doi:10.1145/1165389.945462.

URL http://doi .acm.org/10.1145/1165389.945462

[7] W. Yue, Q. Chen, Dynamic placement of virtual machines with both deterministic and stochastic demands for green cloud computing, Mathematical Problems in Engineering 2014.

[8] J. Zhu, D. Li, J. Wu, H. Liu, Y. Zhang, J. Zhang, Towards bandwidth guarantee in multi-tenancy cloud computing networks, in: Network Protocols (ICNP), 2012 20th IEEE International Conference on, 2012, pp. 1-10. doi:10.1109/ICNP.2012.6459986.

[9] M. Alicherry, T. Lakshman, Network aware resource allocation in distributed clouds, in: INFOCOM, 2012 Proceedings IEEE, IEEE, 2012, pp. 963-971.

[10] X. Li, Z. Qian, S. Lu, J. Wu, Energy efficient virtual machine placement algorithm with balanced and improved resource utilization in a data center, Mathematical and Computer Modelling 58 (5) (2013) 1222-1235.

[11] T. C. Ferreto, M. A. Netto, R. N. Calheiros, C. A. De Rose, Server consolidation with migration control for virtualized data centers, Future Generation Computer Systems 27 (8) (2011) 1027-1034.

[12] T. Wood, P. J. Shenoy, A. Venkataramani, M. S. Yousif, Black-box and gray-box strategies for virtual machine migration., in: NSDI, Vol. 7, 2007, pp. 17-17.

[13] M. H. Ferdaus, M. Murshed, R. N. Calheiros, R. Buyya, Virtual machine consolidation in cloud data centers using aco metaheuristic, in: Euro-Par 2014 Parallel Processing, Springer, 2014, pp. 306-317.

[14] M. Wang, X. Meng, L. Zhang, Consolidating virtual machines with dynamic bandwidth demand in data centers, in: INFOCOM, 2011 Proceedings IEEE, IEEE, 2011, pp. 71-75.

[15] J. Xu, J. A. Fortes, Multi-objective virtual machine placement in virtualized data center environments, in: Green Computing and Communications (GreenCom), 2010 IEEE/ACM Int'l Conference on \& Int'1 Conference on Cyber, Physical and Social Computing (CPSCom), IEEE, 2010, pp. 179-188.

[16] D. Jayasinghe, C. Pu, T. Eilam, M. Steinder, I. Whally, E. Snible, Improving performance and availability of services hosted on iaas clouds with structural constraint-aware virtual machine placement, in: Services Computing (SCC), 2011 IEEE International Conference on, IEEE, 2011, pp. 72-79.

[17] C.-C. Lin, P. Liu, J.-J. Wu, Energy-aware virtual machine dynamic provision and scheduling for cloud computing, in: Cloud Computing (CLOUD), 2011 IEEE International Conference on, IEEE, 2011, pp. 736-737.

[18] L. Grit, D. Irwin, A. Yumerefendi, J. Chase, Virtual machine hosting for networked clusters: Building the foundations for autonomic orchestration, in: Proceedings of the 2nd International Workshop on Virtualization Technology in Distributed Computing, IEEE Computer Society, 2006, p. 7.

[19] J. Békési, G. Galambos, H. Kellerer, A 5/4 linear time bin packing algorithm, Journal of Computer and System Sciences 60 (1) (2000) $145-160$

[20] A. Verma, P. Ahuja, A. Neogi, pmapper: power and migration cost aware application placement in virtualized systems, in: Middleware 2008, Springer, 2008, pp. 243-264.

[21] M. Cardosa, M. R. Korupolu, A. Singh, Shares and utilities based power consolidation in virtualized server environments, in: Integrated Network Management, 2009. IM'09. IFIP/IEEE International Symposium on, IEEE, 2009, pp. 327-334.

[22] M. Bichler, T. Setzer, B. Speitkamp, Capacity planning for virtualized servers, in: Workshop on Information Technologies and Systems (WITS), Milwaukee, Wisconsin, USA, Vol. 1, sn, 2006.

[23] S. Srikantaiah, A. Kansal, F. Zhao, Energy aware consolidation for cloud computing, in: Proceedings of the 2008 conference on Power aware computing and systems, Vol. 10, San Diego, California, 2008.

[24] B. Li, J. Li, J. Huai, T. Wo, Q. Li, L. Zhong, Enacloud: An energy-saving application live placement approach for cloud computing environments, in: Cloud Computing, 2009. CLOUD'09. IEEE International Conference on, IEEE, 2009, pp. 17-24.

[25] A. Verma, P. Ahuja, A. Neogi, Power-aware dynamic placement of hpc applications, in: Proceedings of the 22nd annual international conference on Supercomputing, ACM, 2008, pp. 175-184.

[26] A. Beloglazov, J. Abawajy, R. Buyya, Energy-aware resource allocation heuristics for efficient management of data centers for cloud computing, Future Generation Computer Systems 28 (5) (2012) 755-768.

[27] E. Feller, L. Rilling, C. Morin, Energy-aware ant colony based workload placement in clouds, in: Proceedings of the 2011 IEEE/ACM 12th International Conference on Grid Computing, IEEE Computer Society, 2011, pp. 26-33.

[28] R. Jeyarani, N. Nagaveni, R. V. Ram, Self adaptive particle swarm optimization for efficient virtual machine provisioning in cloud, International Journal of Intelligent Information Technologies (IJIIT) 7 (2) (2011) 25-44.

[29] H. Mi, H. Wang, G. Yin, Y. Zhou, D. Shi, L. Yuan, Online self-reconfiguration with performance guarantee for energy-efficient large-scale cloud computing data centers, in: Services Computing (SCC), 2010 IEEE International Conference on, IEEE, 2010 , pp. 514-521.

[30] J. Xu, J. A. Fortes, Multi-objective virtual machine placement in virtualized data center environments, in: Green Computing and Communications (GreenCom), 2010 IEEE/ACM Int'l Conference on \& Int'l Conference on Cyber, Physical and Social Computing (CPSCom), IEEE, 2010, pp. 179-188. 
[31] Y. Gao, H. Guan, Z. Qi, Y. Hou, L. Liu, A multi-objective ant colony system algorithm for virtual machine placement in cloud computing, Journal of Computer and System Sciences 79 (8) (2013) 1230-1242.

[32] R. Jeyarani, N. Nagaveni, R. Vasanth Ram, Design and implementation of adaptive power-aware virtual machine provisioner (apa-vmp) using swarm intelligence, Future Generation Computer Systems 28 (5) (2012) 811-821.

[33] B. Speitkamp, M. Bichler, A mathematical programming approach for server consolidation problems in virtualized data centers, Services Computing, IEEE Transactions on 3 (4) (2010) 266-278.

[34] J.-W. Lin, C.-H. Chen, C.-Y. Lin, Integrating qos awareness with virtualization in cloud computing systems for delay-sensitive applications, Future Generation Computer Systems 37 (2014) 478-487.

[35] H. N. Van, F. D. Tran, J.-M. Menaud, Performance and power management for cloud infrastructures, in: Cloud Computing (CLOUD), 2010 IEEE 3rd International Conference on, IEEE, 2010, pp. 329-336.

[36] X. Liao, H. Jin, H. Liu, Towards a green cluster through dynamic remapping of virtual machines, Future Generation Computer Systems 28 (2) (2012) 469-477.

[37] S. Chen, J. Wu, Z. Lu, A cloud computing resource scheduling policy based on genetic algorithm with multiple fitness, in: Computer and Information Technology (CIT), 2012 IEEE 12th International Conference on, IEEE, 2012, pp. 177-184.

[38] D. Simon, Biogeography-based optimization, Evolutionary Computation, IEEE Transactions on 12 (6) (2008) $702-713$.

[39] D. Du, D. Simon, Complex system optimization using biogeography-based optimization, Mathematical Problems in Engineering 2013.

[40] F. Hermenier, X. Lorca, J.-M. Menaud, G. Muller, J. Lawall, Entropy: a consolidation manager for clusters, in: Proceedings of the 2009 ACM SIGPLAN/SIGOPS international conference on Virtual execution environments, ACM, 2009, pp. 41-50.

[41] V. Shrivastava, P. Zerfos, K.-W. Lee, H. Jamjoom, Y.-H. Liu, S. Banerjee, Application-aware virtual machine migration in data centers, in: INFOCOM, 2011 Proceedings IEEE, IEEE, 2011, pp. 66-70.

[42] X. Meng, V. Pappas, L. Zhang, Improving the scalability of data center networks with traffic-aware virtual machine placement, in: INFOCOM, 2010 Proceedings IEEE, IEEE, 2010, pp. 1-9.

[43] W. Fang, X. Liang, S. Li, L. Chiaraviglio, N. Xiong, Vmplanner: Optimizing virtual machine placement and traffic flow routing to reduce network power costs in cloud data centers, Computer Networks 57 (1) (2013) 179-196.

[44] A. Karve, T. Kimbrel, G. Pacifici, M. Spreitzer, M. Steinder, M. Sviridenko, A. Tantawi, Dynamic placement for clustered web applications, in: Proceedings of the 15th international conference on World Wide Web, ACM, 2006, pp. 595-604.

[45] X. Shi, H. Jiang, L. He, H. Jin, C. Wang, B. Yu, X. Chen, Developing an optimized application hosting framework in clouds, Journal of Computer and System Sciences 79 (8) (2013) 1214-1229.

[46] I. Giurgiu, C. Castillo, A. Tantawi, M. Steinder, Enabling efficient placement of virtual infrastructures in the cloud, in: Proceedings of the 13th International Middleware Conference, Springer-Verlag New York, Inc., 2012, pp. 332-353.

[47] W. Tian, G. Lu, C. Jing, Y. Zhong, J. Hu, X. Dong, Method and device for implementing load balance of data center resources, uS Patent 8,510,747 (Aug. 13 2013).

[48] S. Srikantaiah, A. Kansal, F. Zhao, Energy aware consolidation for cloud computing, in: Proceedings of the 2008 conference on Power aware computing and systems, Vol. 10, San Diego, California, 2008.

[49] X. Fan, W.-D. Weber, L. A. Barroso, Power provisioning for a warehouse-sized computer, in: ACM SIGARCH Computer Architecture News, Vol. 35, ACM, 2007, pp. 13-23.

[50] E. M. Elnozahy, M. Kistler, R. Rajamony, Energy-efficient server clusters, in: Power-Aware Computer Systems, Springer, 2003 , pp. $179-197$.

[51] C.-H. Lien, Y.-W. Bai, M.-B. Lin, Estimation by software for the power consumption of streaming-media servers, Instrumentation and Measurement, IEEE Transactions on 56 (5) (2007) 1859-1870.

[52] Vmware vsphere 5.5, www.vmware.com/support/vsphere5/doc/vsphere-esx-vcenter-server-55-release-notes.htm (Jan. 2014).

[53] S. K. Langone, Jason, U. S. Alder, Release: Veeam availability suite v8.

[54] D. Simon, M. Ergezer, D. Du, R. Rarick, Markov models for biogeography-based optimization, Systems, Man, and Cybernetics, Part B: Cybernetics, IEEE Transactions on 41 (1) (2011) 299-306.

[55] P. Austin, Cracking the roulette wheel: The system \& story of the cpa who cracked the roulette wheel, createspace independent publishing platform.

[56] H. Ma, An analysis of the equilibrium of migration models for biogeography-based optimization, Information Sciences 180 (18) (2010) 3444-3464.

[57] H. Ma, D. Simon, Analysis of migration models of biogeography-based optimization using markov theory, Engineering Applications of Artificial Intelligence 24 (6) (2011) 1052-1060.

[58] Amazon ec2 instance types. URL http://aws . amazon.com/ec2/instance-types/

[59] Y. Ajiro, A. Tanaka, Improving packing algorithms for server consolidation, in: Int. CMG Conference, 2007, pp. 399-406.

[60] J. Jiang, T. Lan, S. Ha, M. Chen, M. Chiang, Joint vm placement and routing for data center traffic engineering, in: INFOCOM, 2012 Proceedings IEEE, 2012, pp. 2876-2880. doi:10.1109/INFCOM.2012.6195719.

[61] C. Tang, M. Steinder, M. Spreitzer, G. Pacifici, A scalable application placement controller for enterprise data centers, in: Proceedings of the 16th international conference on World Wide Web, ACM, 2007, pp. 331-340.

[62] C. Tian, H. Jiang, A. Iyengar, X. Liu, Z. Wu, J. Chen, W. Liu, C. Wang, Improving application placement for cluster-based web applications, Network and Service Management, IEEE Transactions on 8 (2) (2011) 104-115.

[63] R. Pairault, Z. Yang, S. Krishnan, G. Moore, Utilizing affinity groups to allocate data items and computing resources, uS Patent 8,577,892 (Nov. 5 2013).

[64] A. Gulati, A. Holler, M. Ji, G. Shanmuganathan, C. Waldspurger, X. Zhu, Vmware distributed resource management: Design, implementation, and lessons learned, VMware Technical Journal 1 (1) (2012) 45-64.

[65] Anti-affinity in openstack (2014). URL http://docs.openstack.org/developer/sahara/userdoc/features.html 
[66] J. Lee, Y. Turner, M. Lee, L. Popa, S. Banerjee, J.-M. Kang, P. Sharma, Application-driven bandwidth guarantees in datacenters, in: Proceedings of the 2014 ACM conference on SIGCOMM, ACM, 2014, pp. 467-478.

[67] S. A. Weil, S. A. Brandt, E. L. Miller, D. D. Long, C. Maltzahn, Ceph: A scalable, high-performance distributed file system, in: Proceedings of the 7th symposium on Operating systems design and implementation, USENIX Association, 2006, pp. 307-320.

[68] O. Biran, A. Corradi, M. Fanelli, L. Foschini, A. Nus, D. Raz, E. Silvera, A stable network-aware vm placement for cloud systems, in: Proceedings of the 2012 12th IEEE/ACM International Symposium on Cluster, Cloud and Grid Computing (ccgrid 2012), IEEE Computer Society, 2012, pp. 498-506.

[69] H. Jin, D. Pan, J. Xu, N. Pissinou, Efficient vm placement with multiple deterministic and stochastic resources in data centers, in: Global Communications Conference (GLOBECOM), 2012 IEEE, IEEE, 2012, pp. 2505-2510.

[70] D. Breitgand, A. Epstein, Improving consolidation of virtual machines with risk-aware bandwidth oversubscription in compute clouds, in: INFOCOM, 2012 Proceedings IEEE, IEEE, 2012, pp. 2861-2865.

[71] M. Al-Fares, A. Loukissas, A. Vahdat, A scalable, commodity data center network architecture, in: ACM SIGCOMM Computer Communication Review, Vol. 38, ACM, 2008, pp. 63-74. 
Dr. Qinghua Zheng received his B.S. and M.S. degrees in computer science and technology from Xi'an Jiaotong University in 1990 and 1993, respectively, and his Ph.D. degree in systems engineering from the same university in 1997. He was a postdoctoral researcher at Harvard University in 2002. Since 1995 he has been with the Department of Computer Science and Technology at Xi'an Jiaotong University, and was appointed director of the Department in 2008 and Cheung Kong Professor in 2009. His research interests include intelligent e-learning and network security.

Rui Li is currently a Ph.D. student in the Department of Computer Science and Technology, Xi'an Jiaotong University, China and also a member of MOE Key Lab for Intelligent Networks and Network Security, and also a member of the Satellite-Terrestrial Network Technology R\&D Key Laboratory, Shaanxi Province. He has completed his Bachelor's and Master's degrees from Xi'an Jiaotong University in 2005 and 2009. His research interests include cloud computing and e-learning.

Dr. Xiuqi Li is an Associate Professor at Department of Computer Science and Mathematics in University of North Carolina at Pembroke (UNCP), Pembroke, North Carolina, USA. Prior to joining UNCP, she worked as a senior instructor in Florida Atlantic University, Boca Raton, Florida, USA for five years. She earned Faculty Summer Research Fellowship in 2010. She served as a program committee member and session chair in 20 conferences, a journal reviewer for 10 journals. She was an NSF panelist in 2008. She holds 29 peer-reviewed journal and conference papers. Her research interests include networking, security, multimedia, and web mining. She is a member of ACM and IEEE.

Dr. Nazaraf Shah is a senior lecturer at Coventry University, Coventry, UK. His research interests include intelligent agents, service-oriented computing, web application security and cloud computing. He has over 50 publications in various international conferences and journals.

Dr. Jian-Ke Zhang is currently an associate professor of applied mathematics, Xi'an University of Posts and Telecommunications. He received the B.S. degree in mathematics from Harbin University of Science and Technology in 2001 and the M.S. and $\mathrm{Ph} . \mathrm{D}$. degree in applied mathematics from Xidian University, China in 2006 and 2012, respectively. He current research interests include the development and applications of computational intelligence, robust optimization and interval-valued programming.

Dr. Feng Tian is an associate Professor of Systems Engineering Institute at Xi'an Jiaotong University, China and also is a member of the Satellite-Terrestrial Network Technology R\&D Key Laboratory, Shaanxi Province, and also a member of the MOE 
Key Lab for Intelligent Networks and Network Security. His research interests include system modelling and analysis, cloud computing, Petri nets, and intelligent distance learning environment. He has over 50 publications in books, journals, conference proceedings. He is a member of the IEEE.

Dr. Kuo-Ming Chao is a professor of computing at Coventry University, UK. His research interests include the areas of intelligent agents, service-oriented computing, cloud computing and big data etc as well as their applications such as energy efficiency management and green manufacturing etc. He has over 150 refereed publications in books, journals, conference proceedings. He is a member of the IEEE.

Jia Li is currently a graduate student in the Department of Computer Science and Technology, Xi'an Jiaotong University, China and also a member of MOE Key Lab for Intelligent Networks and Network Security, and also a member of the Satellite-Terrestrial NetworkTechnology R\&D Key Laboratory, Shaanxi Province. He received his Bachelor's degree in Computer Science from Xi'an Jiaotong University in 2013. He is interested in the scheduling and the migration of virtual machines in Cloud Computing. 


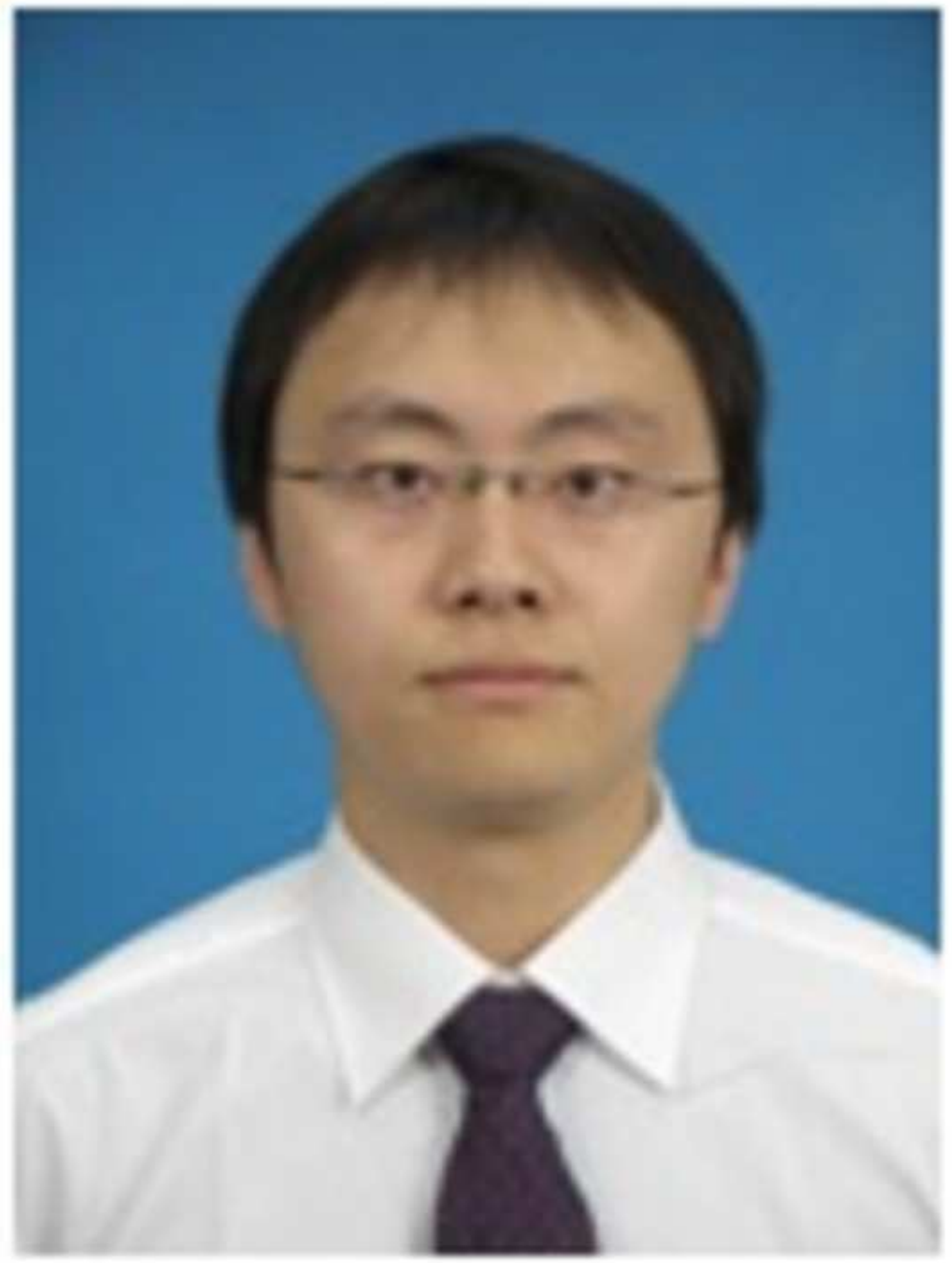




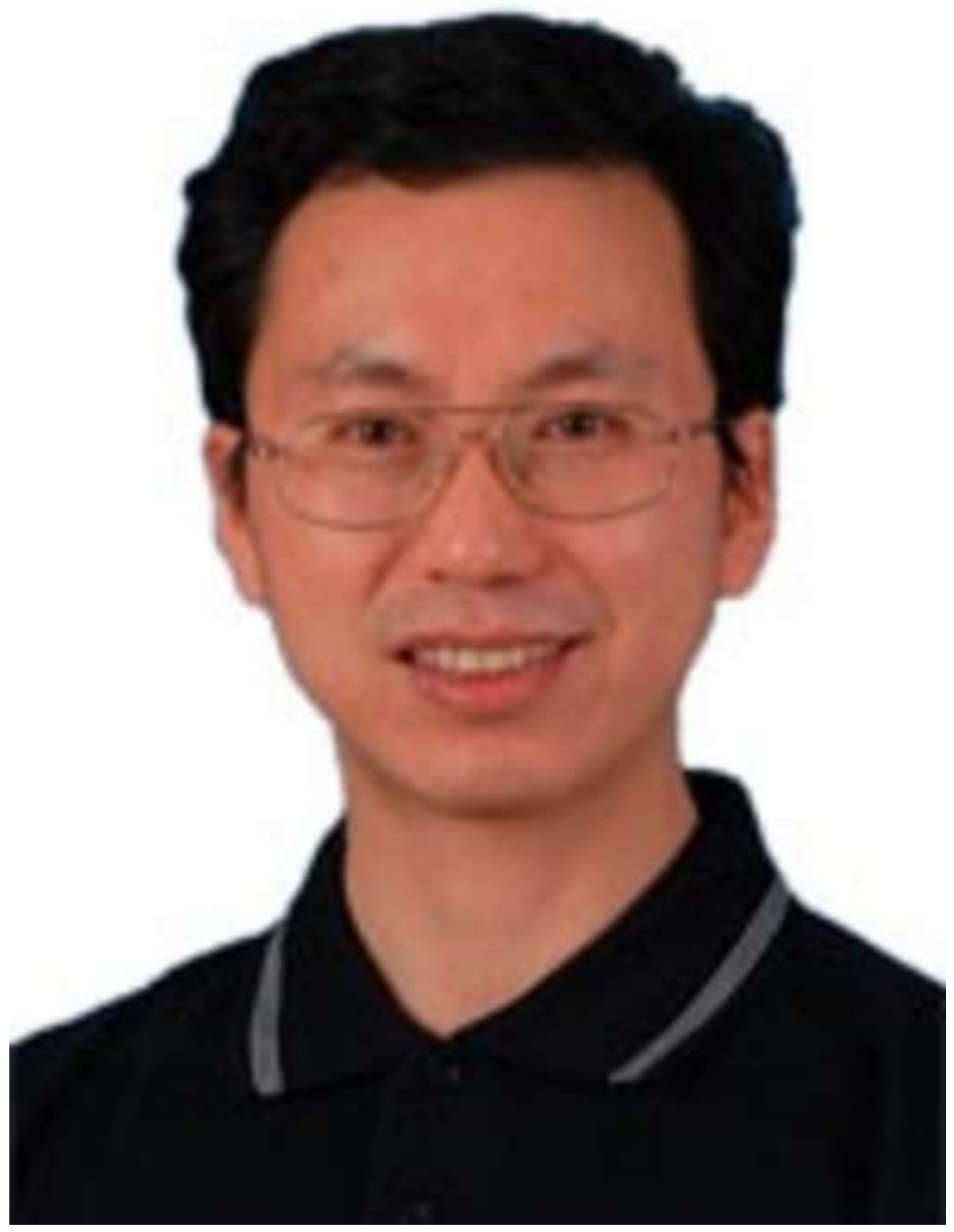




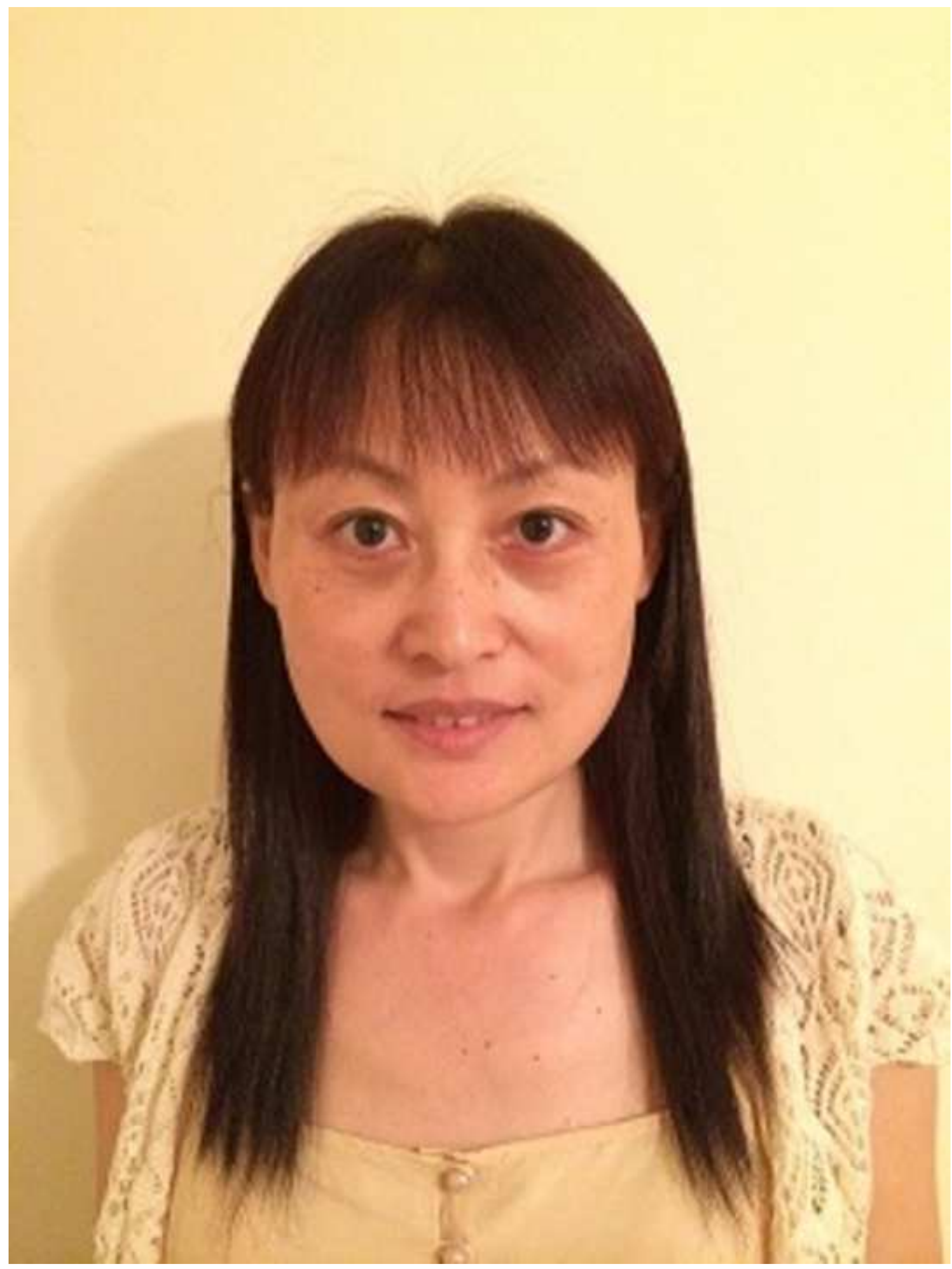




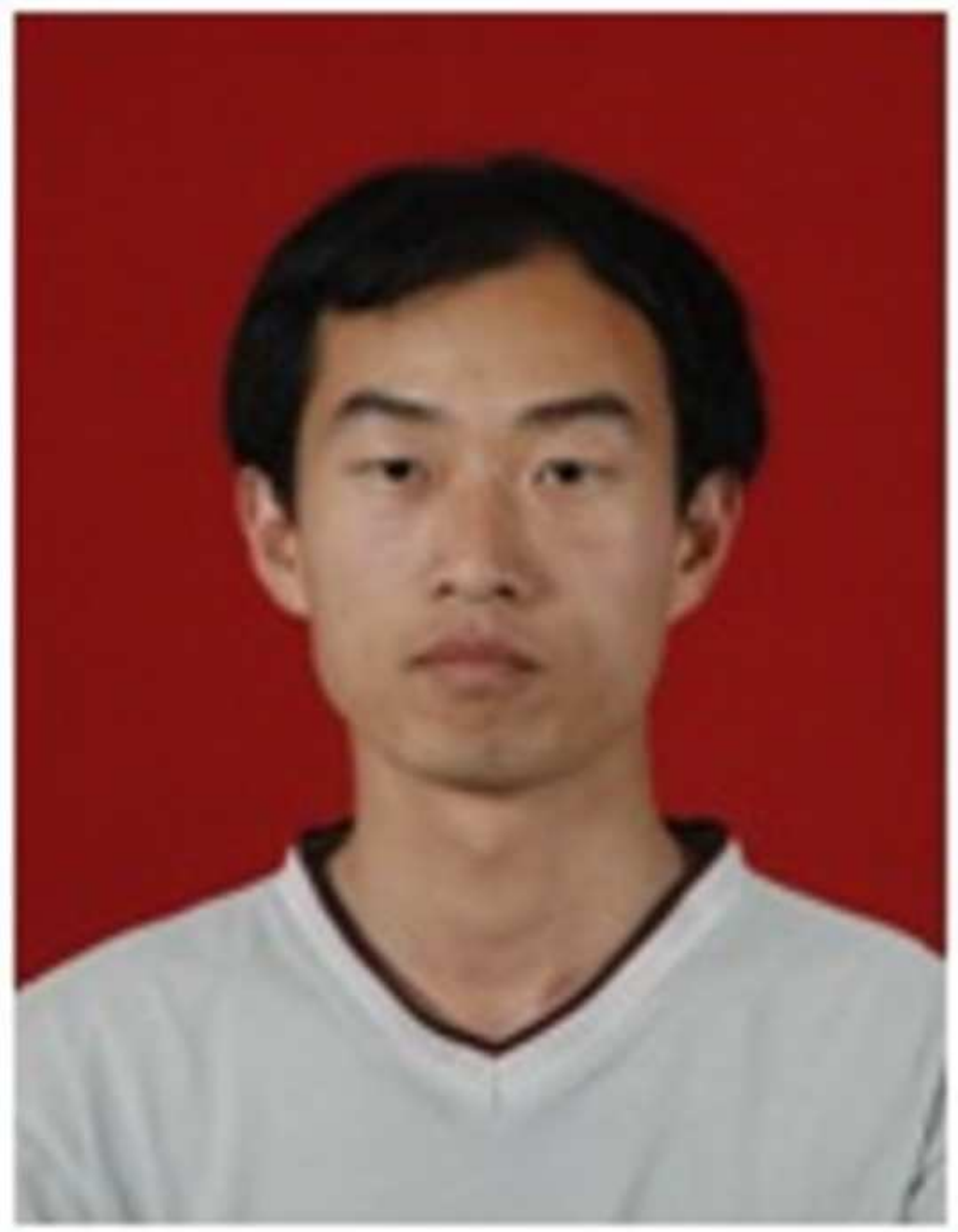




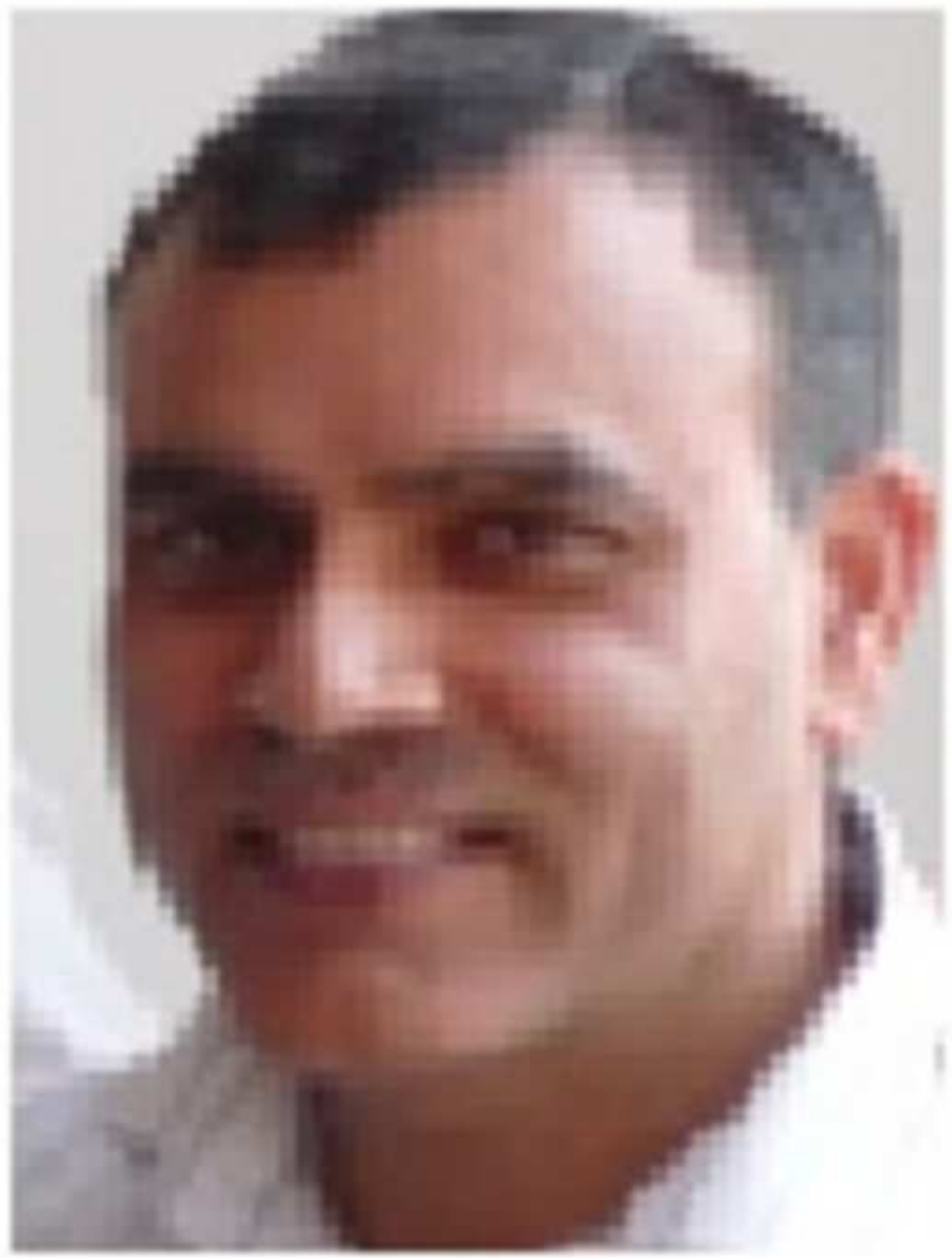




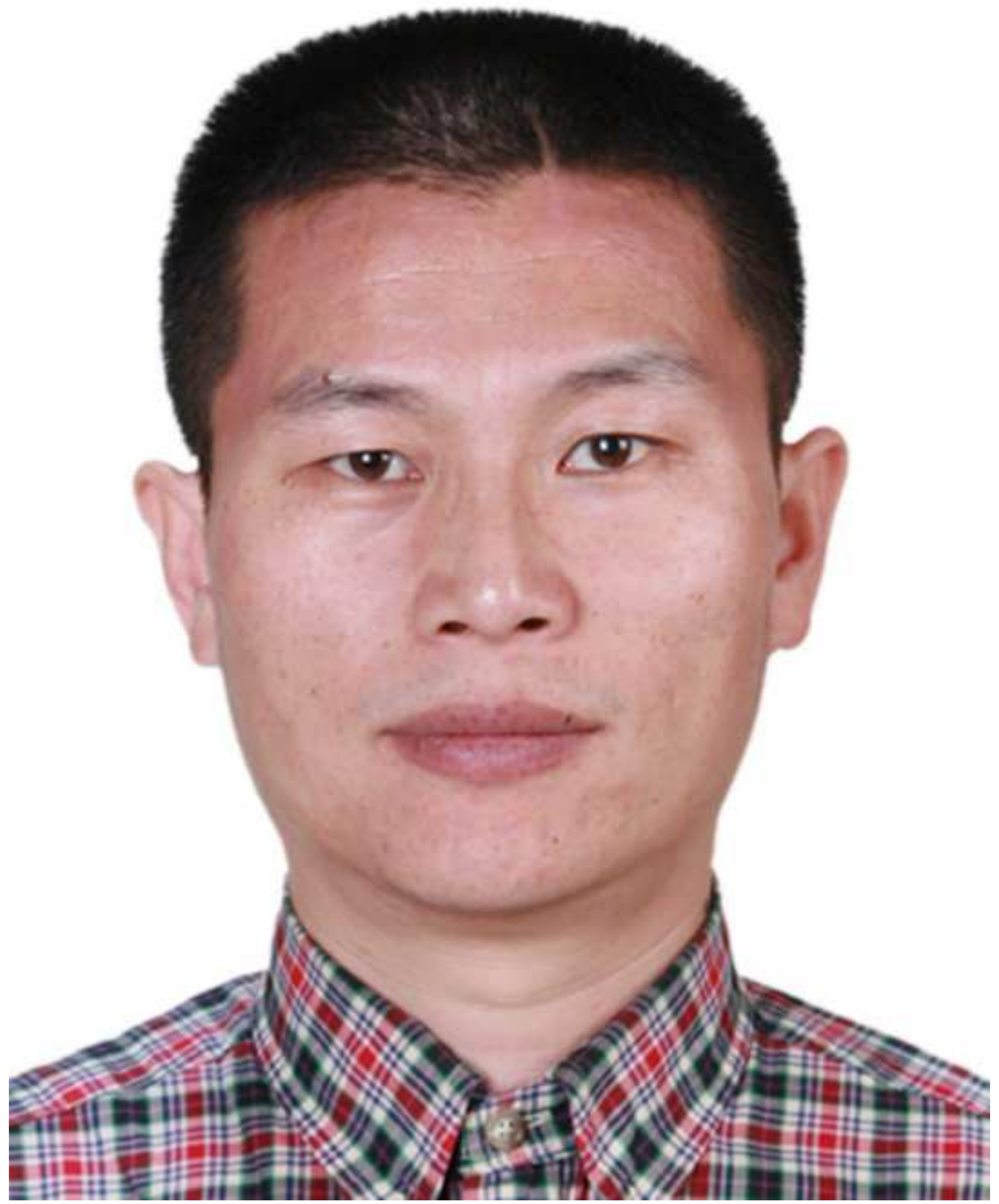




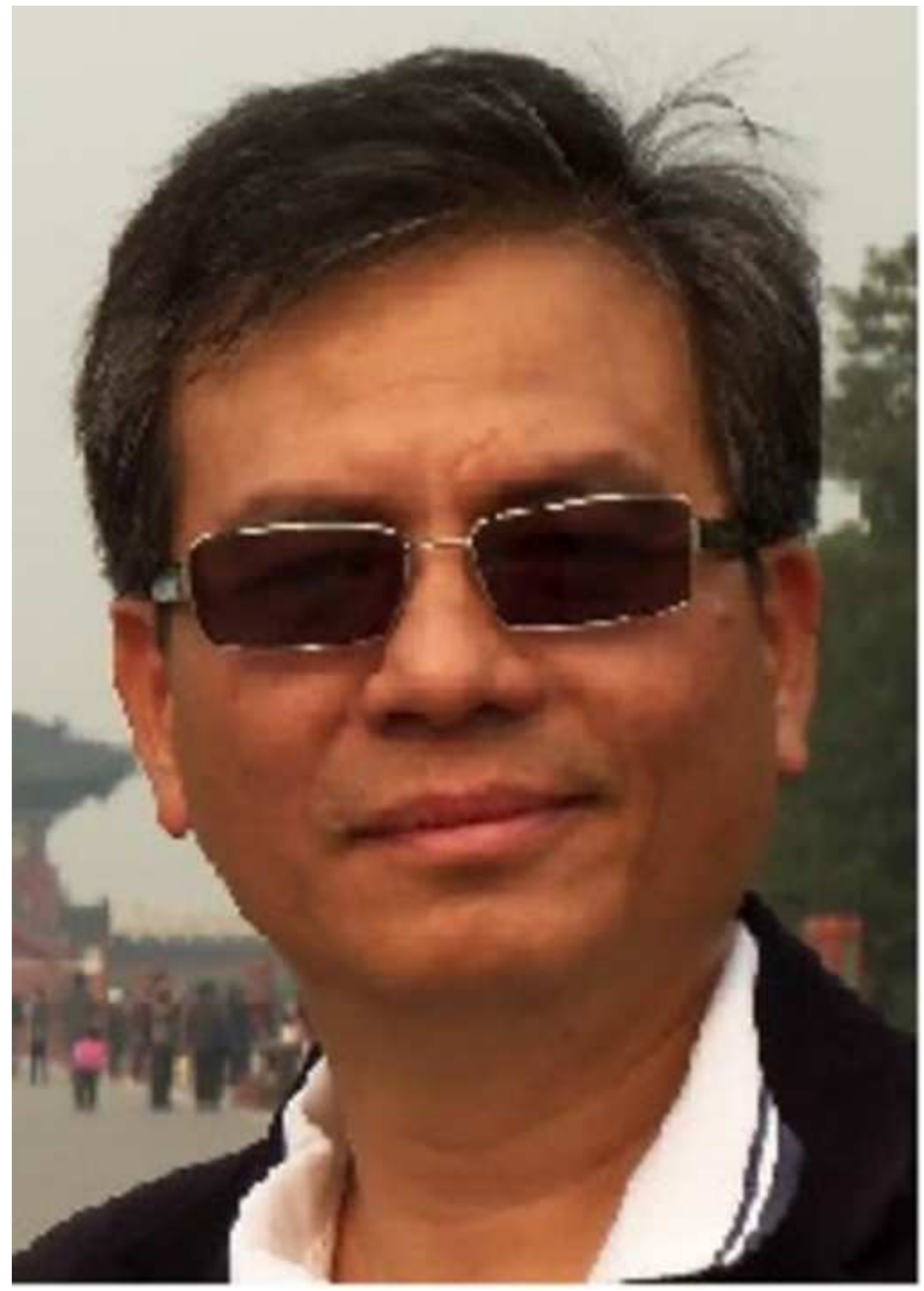




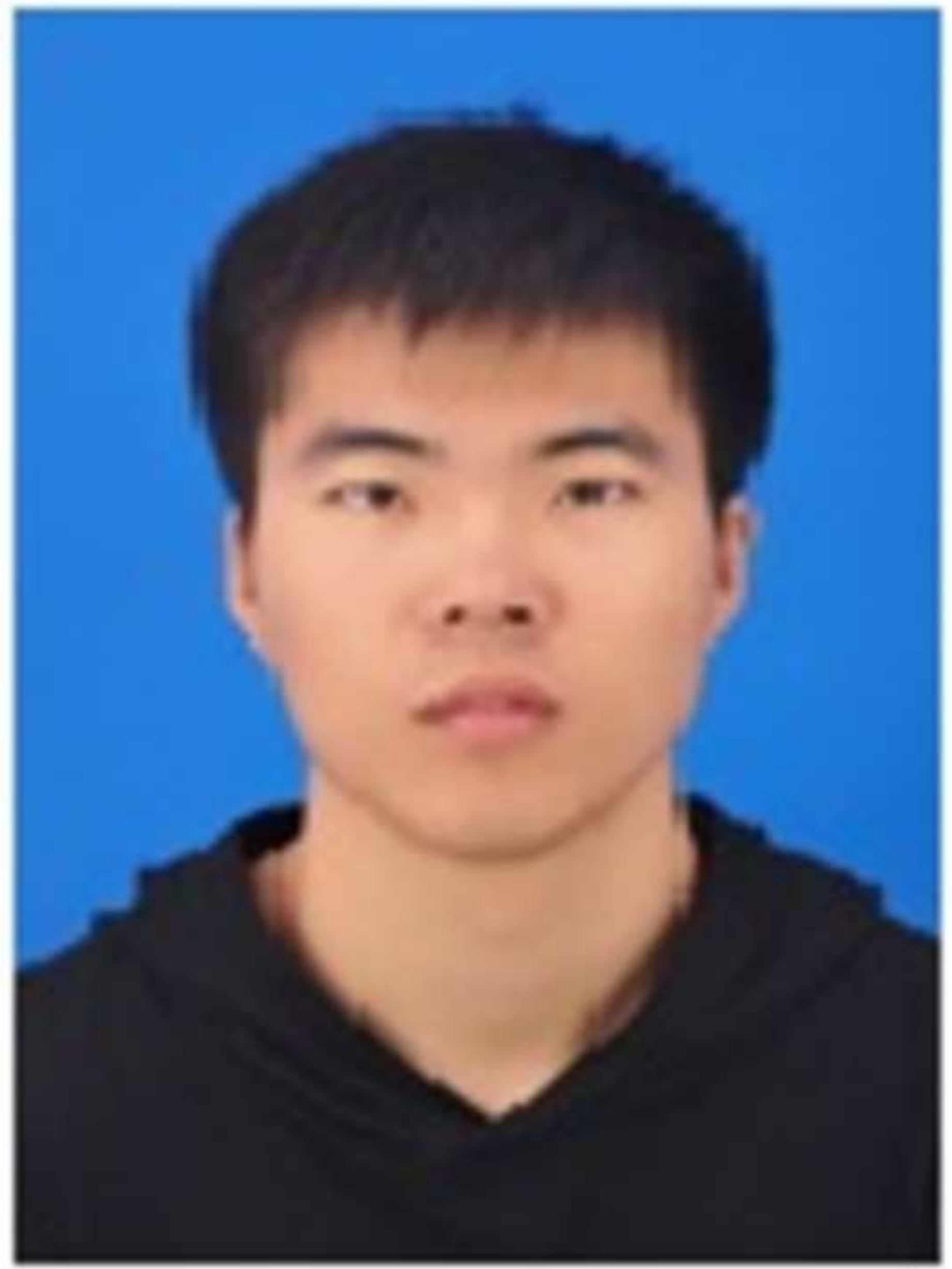

